\title{
Barriers and Facilitators of Engaging Community Health Workers in Non-Communicable Disease (NCD) Prevention and Control in China: A Systematic Review (2006-2016)
}

\author{
Hongfei Long ${ }^{1}$, Wenting Huang ${ }^{1}$, Pinpin Zheng ${ }^{2}$, Jiang $\mathrm{Li}^{2}$, Sha Tao ${ }^{2}$, Shenglan Tang ${ }^{1,3}$ and \\ Abu S. Abdullah 1,3,4,* \\ 1 Global Health Program, Duke Kunshan University, Kunshan 215347, Jiangsu, China; \\ Hongfei.long@dukekunshan.edu.cn (H.L.); wenting@seshglobal.org (W.H.); Shenglan.tang@duke.edu (S.T.) \\ Department of Preventive Medicine, School of Public Health, Fudan University, Shanghai 200032, China; \\ zpinpin@shmu.edu.cn (P.Z.); lijiang_fd@fudan.edu.cn (J.L.); taosha@fudan.edu.cn (S.T.) \\ 3 Duke Global Health Institute, Duke University, Durham, NC 27710, USA \\ 4 Boston University School of Medicine, Boston Medical Center, Boston, MA 02118, USA \\ * Correspondence: abu.abdullah@duke.edu
}

Received: 14 September 2018; Accepted: 23 October 2018; Published: 26 October 2018

check for updates

\begin{abstract}
Background: Non-communicable diseases (NCDs) have become a dominant disease burden in China. Although China has a prevention-centered NCD strategy, the implementation effect in the community has been subjected to manpower and financial difficulties. Engaging community health workers (CHWs) in community-based interventions may be a cost-effective approach to relieve the resource shortage and improve health. This review aimed to synthesize evidence on types of NCD-related care that was provided by CHWs in China, and to identify relevant barriers and facilitators. Methods: A literature search was conducted in Medline, PubMed, ProQuest, and Google Scholar databases for English-written, peer-reviewed articles published from 1996 to 2016 that reported findings from NCD-related interventions delivered by CHWs in China. Each article was extracted independently by two researchers. Results: Twenty distinct studies met the inclusion criteria. The two most common types of CHW-led NCD-related care were diabetes and hypertension management $(n=7)$ and mental health care $(n=7)$. Thirteen studies discussed the barriers and 16 studies reported facilitators. The most common barriers included lack of support $(n=6)$, lack of resources $(n=4)$, and heavy reliance on technology $(n=4)$. The common facilitators included an integrated health system $(n=9)$, community and patient trust $(n=5)$, high quality training $(n=5)$, and CHWs' capacity $(n=5)$. Fourteen studies mentioned training content, while only eight described detailed procedures and duration. Conclusions: This review suggests that trained and supervised Chinese CHWs had the capacity to provide grassroots NCDs preventive interventions. In order to increase the generalizability and sustainability of such programs, studies with robust designs are needed to explore the effectiveness of $\mathrm{CHW}$-led programs, and the intervention strategies to improve the practice of CHWs in various settings.
\end{abstract}

Keywords: community health workers; community-based intervention; non-communicable diseases; China; systematic review

\section{Introduction}

In the latest decade, chronic non-communicable diseases (NCDs) have become a dominant disease burden. In 2005 in China, 80\% of deaths and 70\% of disability-adjusted life-years lost (DALYs) were 
attributed to NCDs [1,2]. Many people suffering from NCDs remain untreated due to inadequate attention and funds from the governments [3]. Meanwhile, as China has seen economic growth with its population aging, lifestyle changes, and socioeconomic transition, the prevalence and impact of this NCD epidemic are likely to expand over time [4].

To curb this epidemic, China has implemented a series of nationwide community-based NCD prevention and control programs, including hypertension screening, counseling, salt intake reduction education, healthy lifestyle promotion, and smoking cessation [5]. Most of these programs have been delivered to community members by different categories of public health workers at primary care level (e.g., doctors and nurses in county-level clinics and Centers for Disease Control). However, there are still needs to mobilize other sustainable human resources at community level, especially in remote rural areas, to reach the public with wide categories of evidence-based NCD prevention and control efforts.

Evidence shows that engaging community health workers (CHWs) in community-based health care programs can relieve the health professional shortage and improve the population health in a cost-saving manner [6]. The World Health Organization (WHO) defines CHWs as the public health workers who are selected by community members, and who live in the communities they serve [7]. However, as CHWs are given different names from their assigned tasks associated with local cultures and health systems (e.g., traditional birth attendants, community health volunteers, village health workers, etc.), there is no any universal title for all CHWs [8]. For example, in China, CHWs were initially named "barefoot doctors" from the 1950s to 1980s [8]. Since then, in the 1981 state healthcare reform, the barefoot doctors have been entitled as "village doctors", if they passed the national qualification exam after receiving basic medical training [9]. Usually, village doctors receive three years of junior college education and one year of in-service training in a medical practice. They also have to receive continuing education of 15 credit hours (accounting for 45-90 class hours) per year. We will use the term "CHWs" to describe all these categories of healthcare workers in this paper, unless specified otherwise.

In recent decades, Chinese CHWs have been actively engaging themselves in NCD prevention and control services. According to the China Ministry of Health, NCD-related community-based health services should include elderly health management, case management for hypertension, type II diabetes, and mental illnesses [10]. Recent policy reforms have shown a strong interest in shifting chronic disease management tasks to village doctors [11]. Moreover, the literature has revealed that with proper training, support, and supervision, CHWs can deliver services such as diabetes screenings, cardiovascular diseases case management, healthy lifestyle counseling, early detection, referral for priority chronic diseases, and medication compliance management in NCD programs [12-15]. However, these have not been officially included in the $\mathrm{CHWs}^{\prime}$ responsibilities and agendas.

Given the huge potential for CHWs in their contributions to NCD prevention and control in China, there is a need to generate an efficient and feasible strategy to strengthen their roles in addressing NCDs in an organized manner. Nevertheless, there has been limited literature that summarized the range of NCD programs led by CHWs in China, or that examined the challenges and facilitators encountered by CHWs when delivering NCD-related preventive services. As China is working to implement the Medium-to-Long Term Plan for the Prevention and Treatment of Chronic Diseases (2017-2025), the findings from this systematic review can and will inform policy and public health interventions surrounding community health workers in China. Therefore, the purposes of this review were as follows: (1) to document the types of NCD-related healthcare or preventive services that were delivered by CHWs in China; and (2) to summarize the barriers and facilitators for engaging CHWs in NCDs prevention and control at primary care level in China. This systematic review will be guided by the following research question: What are the types of NCD related health programs provided by CHWs in China as reported in studies from 1996 to 2016, and what are the barriers and facilitating factors? 


\section{Methodology}

\subsection{Data Sources}

This systematic review investigated all the English language, peer-reviewed journal publications that entirely or partially focused on the roles of CHWs in common NCD prevention and control programs in China.

We started this review by searching the published journal articles on Medline, ProQuest, Google Scholar, and Scopus, using text words 'community health workers', 'village doctors', 'non-communicable diseases (NCDs)', and 'China', as well as corresponding medical subject headings (MeSHs) terms. We also searched for non-MeSH terms such as 'lay health workers', 'lay health supporters', 'community health promoters', 'community', 'rural', and 'chronic', as well as their synonyms in English to expand the inclusion of available studies. Meanwhile, we also checked the reference lists of the identified articles to explore additional publications to broaden the inclusion criteria.

\subsection{Study Selection}

We targeted articles that were written in the English language, with interventions conducted in China and published in the last 20 years (1996-2016). We used the publication date instead of the study date, as the publication date is relatively more accessible.

The inclusion and exclusion criteria for CHWs-delivered studies followed the PICOS framework [16] as follows:

1. Participants: Participants can be patients suffering from the aforementioned NCDs, NCD high-risk populations, or the general populations without restricting types of participants, because preventive services and interventions can reach different populations based on specific needs and settings.

2. Intervention types: NCD-related preventive measures or health promotion interventions that were provided by CHWs, village doctors, lay health supporters, or those healthcare personnel who delivered community-based services but received less formal training than health professionals.

3. Comparison: Not applicable.

4. Outcome: Delivery of reported intervention, provider types, categories of NCD topics, and challenges and facilitators.

5. Study types: Both descriptive and evaluation studies conducted in China focusing on community-based NCD-related programs (including programs focusing on the chronic diseases as well as the NCD risk factors), such as hypertension screening, physical activity promotions, early detection for cardiovascular diseases (CVDs), diabetes, and mental health illness.

The exclusion criteria included the following:

1. Articles that did not focus on China.

2. Articles that focused on the health professionals (physicians, doctors, nurses) rather than CHWs as we have defined for this review.

3. Articles that mainly discussed general primary health care (i.e., non-NCD related services), such as immunization, infectious disease case management, family planning, reproductive health, environmental health, and maternal and child health.

4. Articles that did not describe structured NCD-related interventions (e.g., news, conference reports, books, reviews, health system analysis, disease prevalence).

Two members of our research team (W.H. and H.L.) independently assessed the relevant studies based on the inclusion and exclusion criteria. Each reviewer scanned the titles and abstracts of all potential articles to determine eligibilities. Disagreements were resolved consensually, first by these 
two members and in some cases with inputs from the project leader (A.S.A.). The study selection process in this review followed the PRISMA flow diagram [17] and is summarized in Figure 1.

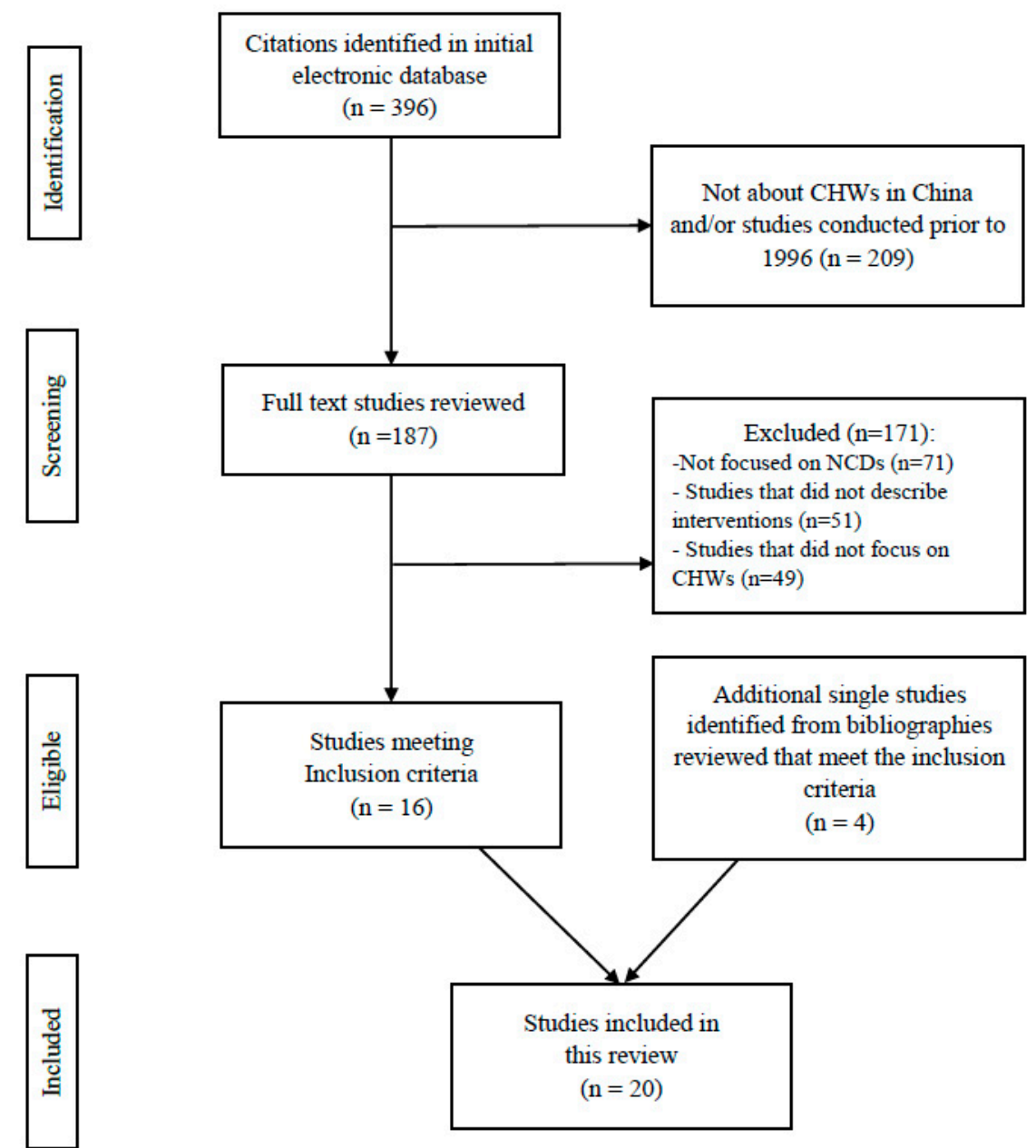

Figure 1. Selection process for identifying relevant studies. NCDs, non-communicable diseases; CHWs, community health workers.

We then read the full texts of all eligible materials and summarized relevant content. Using an Excel form, we assigned each eligible article with a unique reference number and extracted the following information: the types of program, titles of CHWs, the services provided by or/and the responsibilities of $\mathrm{CHWs}$, program duration, training received by $\mathrm{CHWs}$, and challenges and facilitators faced by CHWs in the engaged program. We also summarized the training types received by CHWs as well as the training duration, if this information was available (Table 1). 
Table 1. Description of non-communicable disease (NCD)-related studies and programs that engaged community health workers (CHWs) in China.

\begin{tabular}{|c|c|c|c|c|c|c|c|c|c|c|c|c|c|}
\hline \multirow{2}{*}{ No. } & \multirow{2}{*}{ Author } & \multirow{2}{*}{$\begin{array}{c}\text { Year; } \\
\text { Location }\end{array}$} & \multirow{2}{*}{$\begin{array}{l}\text { Type of } \\
\text { NCDs }\end{array}$} & \multirow{2}{*}{$\begin{array}{l}\text { Provider } \\
\text { Type }\end{array}$} & \multirow{2}{*}{$\begin{array}{c}\text { Payment } \\
\text { Mechanism/ } \\
\text { Structure } \\
\text { (Paid/Unpaid) }\end{array}$} & \multicolumn{7}{|c|}{ Indicators } & \multirow{2}{*}{$\begin{array}{l}\text { Key Findings/ } \\
\text { Lessons Learned }\end{array}$} \\
\hline & & & & & & Types of Services & $\begin{array}{l}\text { Program } \\
\text { Duration }\end{array}$ & $\begin{array}{l}\text { Ended/ } \\
\text { Current }\end{array}$ & Types of Training & $\begin{array}{l}\text { Training } \\
\text { Duration }\end{array}$ & Challenges $(-)$ & $\begin{array}{l}\text { Facilitated Factors } \\
\qquad(+)\end{array}$ & \\
\hline \multicolumn{14}{|c|}{ Diabetes and/or hypertension } \\
\hline 1 & $\begin{array}{l}\text { Feng et } \\
\text { al. [18] }\end{array}$ & $\begin{array}{c}2013 ; \\
\text { Lu'An, } \\
\text { An'hui } \\
\text { Province }\end{array}$ & T2DM & VDs & paid & $\begin{array}{l}\text { 1. Glucose } \\
\text { screening; } \\
\text { 2. measured body } \\
\text { weight and blood } \\
\text { pressure; } \\
\text { 3. counseling on } \\
\text { glucose screening; } \\
\text { 4. promoted } \\
\text { screening } \\
\text { participation via } \\
\text { biannual follow-up } \\
\text { glucose screening; } \\
\text { 5. referral; } \\
\text { 6. behavioral change } \\
\text { counseling for } \\
\text { pre-diabetics }\end{array}$ & $\begin{array}{l}6 \text { years (every } \\
12 \text { months for } \\
\text { plasma glucose } \\
\text { and ever month } \\
\text { for body weight } \\
\text { and blood } \\
\text { pressure) }\end{array}$ & Current & $\begin{array}{l}\text { Web-based training, } \\
\text { with an } \\
\text { 'occupational toolkit' } \\
\text { consisting of a } \\
\text { workbook, a manual } \\
\text { guidance, and a set } \\
\text { of cue-cards. For } \\
\text { example, each } \\
\text { cue-card enlisted } \\
\text { critical steps or } \\
\text { elements for } \\
\text { delivering a specific } \\
\text { type of counseling; } \\
\text { the manual was a } \\
\text { reference book } \\
\text { including } \\
\text { elementary } \\
\text { protocols on } \\
\text { common problems } \\
\text { and solution tips for } \\
\text { diabetes prevention }\end{array}$ & $\mathrm{N} / \mathrm{A}$ & $\begin{array}{l}\text { 1. Most VDs were } \\
\text { unaware of diabetes } \\
\text { prevention; } \\
\text { 2. already heavy } \\
\text { workload; } 3 \text {. heavy } \\
\text { reliance on } \\
\text { electronic/commuter } \\
\text { support may } \\
\text { beyond the ability of } \\
\text { VDs' and elder } \\
\text { villagers' in rural } \\
\text { areas }\end{array}$ & $\begin{array}{l}\text { 1. Trust from } \\
\text { patients and } \\
\text { communities; } \\
\text { 2. service was } \\
\text { simple, capable for } \\
\text { VDs (only } 15 \\
\text { minutes); } 3 \text {. } \\
\text { well-established } \\
\text { guidelines and } \\
\text { manuals; } 4 \text {. village } \\
\text { clinics was } \\
\text { appropriate setting; } \\
\text { 5. electronic support } \\
\text { and web-based } \\
\text { training were } \\
\text { cost-saving and time } \\
\text { flexible; } 6 \\
\text { performance-based } \\
\text { incentives; } 7 \text {. local } \\
\text { health authorities } \\
\text { support on } \\
\text { resources }\end{array}$ & $\begin{array}{l}\text { The study examined } \\
\text { key success factors } \\
\text { in a synergistic way } \\
\text { towards integrated } \\
\text { and sustainable } \\
\text { diabetes prevention. } \\
\text { It introduced a } \\
\text { package of } \\
\text { long-term incentives } \\
\text { and continuous } \\
\text { capacity building } \\
\text { mechanism. It built } \\
\text { up a potential } \\
\text { operational cycle for } \\
\text { catalyzing similar } \\
\text { efforts in the local } \\
\text { prefecture }\end{array}$ \\
\hline 2 & $\begin{array}{l}\text { Lin et al. } \\
\text { [19] }\end{array}$ & $\begin{array}{l}\text { 2014;Xilingol } \\
\text { county; } \\
\text { Inner } \\
\text { Mongolia }\end{array}$ & $\begin{array}{l}\text { Hypertension; } \\
\text { Diabetes }\end{array}$ & VDs & paid & $\begin{array}{l}\text { 1. Case } \\
\text { management and } \\
\text { monitoring via EHR; } \\
\text { 2. regular } \\
\text { followed-up check } \\
\text { on medication } \\
\text { compliance; } \\
\text { 3. measured blood } \\
\text { pressure and blood } \\
\text { sugar levels }\end{array}$ & 2008-2011 & Ended & N/A & N/A & $\begin{array}{l}\text { Lack of policy } \\
\text { support from the } \\
\text { health system }\end{array}$ & $\begin{array}{l}\text { Close connection } \\
\text { with higher levels of } \\
\text { the healthcare } \\
\text { system, if } \\
\text { implemented in } \\
\text { large-scale }\end{array}$ & $\begin{array}{l}\text { Cloud-based EHR } \\
\text { had the potential to } \\
\text { improve care } \\
\text { provision in rural } \\
\text { China and efficiency } \\
\text { of disease } \\
\text { management. }\end{array}$ \\
\hline 3 & $\begin{array}{l}\text { Chen et } \\
\text { al. [20] }\end{array}$ & $\begin{array}{l}\text { 2014; Lu } \\
\text { An; Anhui } \\
\text { Province }\end{array}$ & T2DM & VDs & paid & $\begin{array}{l}\text { 1. Identified } \\
\text { high-risk patients; } \\
\text { 2. case follow-up } \\
\text { counseling and } \\
\text { health education on } \\
\text { diabetes risks, } \\
\text { balanced diets, and } \\
\text { physical activities }\end{array}$ & $\begin{array}{l}6 \text { months; } 1 \\
\text { month/session }\end{array}$ & Ended & $\begin{array}{l}\text { Instructions on the } \\
\text { application method } \\
\text { of the program, with } \\
\text { standardized } \\
\text { "step-by-step" } \\
\text { navigation for VDs } \\
\text { to follow in practice }\end{array}$ & $\mathrm{N} / \mathrm{A}$ & $\begin{array}{l}\text { 1. Lack of electricity } \\
\text { security (facility) in } \\
\text { remote settings; } \\
\text { 2. communication } \\
\text { difficulties: } \\
\text { sometimes unable to } \\
\text { engage patients to } \\
\text { finish all the } \\
\text { required } \\
\text { interventions }\end{array}$ & $\begin{array}{l}\text { 1. Easy to follow the } \\
\text { navigation; } \\
\text { 2. professional } \\
\text { knowledge built in } \\
\text { the application } \\
\text { helps case } \\
\text { identification and } \\
\text { management; } \\
\text { 3. high acceptance } \\
\text { rate among patients }\end{array}$ & $\begin{array}{l}\text { The web-based tool } \\
\text { SWAP-DM2 can } \\
\text { increase the } \\
\text { effectiveness of } \\
\text { T2DM preventive } \\
\text { services delivered } \\
\text { by VDs, and may } \\
\text { increase the frontier } \\
\text { clinicians' } \\
\text { participation in } \\
\text { diabetes prevention } \\
\text { in similar settings. }\end{array}$ \\
\hline
\end{tabular}


Table 1. Cont.

\begin{tabular}{|c|c|c|c|c|c|c|c|c|c|c|c|c|c|}
\hline \multirow{2}{*}{ No. } & \multirow{2}{*}{ Author } & \multirow{2}{*}{$\begin{array}{c}\text { Year; } \\
\text { Location }\end{array}$} & \multirow{2}{*}{$\begin{array}{l}\text { Type of } \\
\text { NCDs }\end{array}$} & \multirow{2}{*}{$\begin{array}{l}\text { Provider } \\
\text { Type }\end{array}$} & \multirow{2}{*}{$\begin{array}{c}\text { Payment } \\
\text { Mechanism// } \\
\text { Structure } \\
\text { (Paid/Unpaid) }\end{array}$} & \multicolumn{7}{|c|}{ Indicators } & \multirow{2}{*}{$\begin{array}{l}\text { Key Findings/ } \\
\text { Lessons Learned }\end{array}$} \\
\hline & & & & & & Types of Services & $\begin{array}{l}\text { Program } \\
\text { Duration }\end{array}$ & $\begin{array}{l}\text { Ended/ } \\
\text { Current }\end{array}$ & Types of Training & $\begin{array}{c}\text { Training } \\
\text { Duration } \\
\end{array}$ & Challenges (-) & $\begin{array}{l}\text { Facilitated Factors } \\
\qquad(+)\end{array}$ & \\
\hline 4 & $\begin{array}{l}\text { Zhong et } \\
\text { al. [21] }\end{array}$ & $\begin{array}{c}\text { 2015; } \\
\text { Tonglin, } \\
\text { Hefei, } \\
\text { Bangbu, } \\
\text { Anhui } \\
\text { Province }\end{array}$ & Diabetes & $\begin{array}{c}\text { Peer } \\
\text { Leaders; } \\
\text { CHSC Staff }\end{array}$ & $\mathrm{N} / \mathrm{A}$ & $\begin{array}{l}\text { Bi-weekly } \\
\text { educational } \\
\text { meetings co-led by } \\
\text { PLs and CHS staffs. } \\
\text { Topics: diets, } \\
\text { physical activities, } \\
\text { medications, foot } \\
\text { care, stress } \\
\text { management. PLs' } \\
\text { responsibilities: } \\
\text { outreach, } \\
\text { promotions, } \\
\text { emotional support, } \\
\text { and non- } \\
\text { professional } \\
\text { activities (Tai Chi, } \\
\text { morning exercise, } \\
\text { etc.) }\end{array}$ & $\begin{array}{l}6 \text { months/ } \\
\text { session }\end{array}$ & $\mathrm{N} / \mathrm{A}$ & $\mathrm{N} / \mathrm{A}$ & $\mathrm{N} / \mathrm{A}$ & $\begin{array}{l}\text { Lack of staff } \\
\text { resources in } \\
\text { sub-communities }\end{array}$ & $\begin{array}{l}\text { 1. Close relationship } \\
\text { with peer leaders; } \\
\text { 2. knowledge; } \\
\text { 3. high patient } \\
\text { engagement }\end{array}$ & $\begin{array}{l}\text { The PLSP was } \\
\text { effective for } \\
\text { subcommunity-based } \\
\text { diabetes } \\
\text { self-management. } \\
\text { May be } \\
\text { generalizable to } \\
\text { cardiovascular } \\
\text { prevention. }\end{array}$ \\
\hline 5 & $\begin{array}{l}\text { Li et al. } \\
\text { [22] }\end{array}$ & $\begin{array}{l}\text { 2015; three } \\
\text { provinces } \\
\text { in China, } \\
\text { specific } \\
\text { location } \\
\text { not } \\
\text { mentioned }\end{array}$ & $\begin{array}{l}\text { Hypertension; } \\
\text { Diabetes }\end{array}$ & $\begin{array}{l}\text { village } \\
\text { doctor }\end{array}$ & paid & $\begin{array}{l}\text { 1. Hypertension } \\
\text { and/or diabetes } \\
\text { case management; } \\
\text { 2. created citizen } \\
\text { health record (no } \\
\text { specificinformation } \\
\text { provided for this } \\
\text { category) }\end{array}$ & $\begin{array}{l}2014 \\
\text { (cross-sectional } \\
\text { survey among } \\
\text { VDs) }\end{array}$ & Ended & $\begin{array}{l}\text { Routine training } \\
\text { programs including } \\
\text { content such as } \\
\text { health care policy; } \\
\text { standards; BPHS } \\
\text { quality } \\
\text { management; and } \\
\text { the norms, } \\
\text { standards, and } \\
\text { service delivery } \\
\text { paths of BPHS. }\end{array}$ & N/A & $\begin{array}{l}\text { 1. Limited } \\
\text { compensation and } \\
\text { low financial } \\
\text { incentive; } 2 \text {. Uneven } \\
\text { geographic coverage } \\
\text { of the NCMS } \\
\text { insurance contract }\end{array}$ & $\begin{array}{l}\text { 1. Education and } \\
\text { training } \\
\text { opportunities, } \\
\text { 2. public health care } \\
\text { subsidy; } \\
\text { 3. integrated } \\
\text { management and } \\
\text { supervision; } \\
\text { 4. access to NCMS } \\
\text { insurance program } \\
\text { contract }\end{array}$ & $\begin{array}{l}\text { Increasing subsidies } \\
\text { for VDs, availability } \\
\text { and attendance of } \\
\text { training } \\
\text { opportunities, } \\
\text { integrated } \\
\text { management, and } \\
\text { NCMS contracting } \\
\text { of village clinics } \\
\text { were important } \\
\text { factors in increasing } \\
\text { BPHS provision in } \\
\text { rural areas. }\end{array}$ \\
\hline 6 & $\begin{array}{l}\text { Browning } \\
\text { et al. [13] }\end{array}$ & $\begin{array}{l}\text { 2016; } \\
\text { Fengtai } \\
\text { District, } \\
\text { Beijing }\end{array}$ & T2DM & $\begin{array}{l}\text { Health } \\
\text { coach } \\
\text { (health } \\
\text { workers } \\
\text { from the } \\
\text { local CHS) }\end{array}$ & paid & $\begin{array}{l}\text { Bi-weekly/monthly } \\
\text { telephone and } \\
\text { face-to-face } \\
\text { motivational } \\
\text { interviews (MIs), to } \\
\text { provide } \\
\text { psychosocial } \\
\text { support and lifestyle } \\
\text { counseling to } \\
\text { improve the } \\
\text { outcome of glycemic } \\
\text { control and self-care } \\
\text { of T2DM patients. }\end{array}$ & 1 year & Current & $\begin{array}{l}\text { Key concepts in } \\
\text { patient-centered } \\
\text { communinications, } \\
\text { health psychology, } \\
\text { epidemiology of } \\
\text { targeted conditions, } \\
\text { the framework and } \\
\text { rationale of MIs, } \\
\text { and the application } \\
\text { of MIs core sillls. } \\
\text { Review workshops } \\
\text { were arranged one } \\
\text { month after the } \\
\text { project initiation, } \\
\text { and every three } \\
\text { months after that. }\end{array}$ & $\begin{array}{l}\text { Initial } \\
\text { training: } 20 \\
\text { hours; } \\
\text { review } \\
\text { workshop: } \\
\text { half day per } \\
\text { workshop }\end{array}$ & $\begin{array}{l}\text { Not generalizable to } \\
\text { rural settings with } \\
\text { limited human } \\
\text { resources }\end{array}$ & $\begin{array}{l}\text { 1. Good learning } \\
\text { and practice } \\
\text { capacity; } \\
\text { 2. well-organized } \\
\text { training process } \\
\text { including review } \\
\text { workshops }\end{array}$ & $\begin{array}{l}\text { The result of this } \\
\text { study indicated that } \\
\text { the coach-led } \\
\text { psychological } \\
\text { intervention had the } \\
\text { potential to improve } \\
\text { mental health status } \\
\text { and well-being of } \\
\text { T2DM patients. The } \\
\text { intervention could } \\
\text { provide evidence for } \\
\text { the establishment of } \\
\text { regular and free } \\
\text { clinical health } \\
\text { checks for people } \\
\text { with T2DM. }\end{array}$ \\
\hline
\end{tabular}


Table 1. Cont.

\begin{tabular}{|c|c|c|c|c|c|c|c|c|c|c|c|c|c|}
\hline \multirow{2}{*}{ No. } & \multirow{2}{*}{ Author } & \multirow{2}{*}{$\begin{array}{c}\text { Year; } \\
\text { Location }\end{array}$} & \multirow{2}{*}{$\begin{array}{l}\text { Type of } \\
\text { NCDs }\end{array}$} & \multirow{2}{*}{$\begin{array}{l}\text { Provider } \\
\text { Type }\end{array}$} & \multirow{2}{*}{$\begin{array}{l}\text { Payment } \\
\text { Mechanism/ } \\
\text { Structure } \\
\text { (Paid/Unpaid) }\end{array}$} & \multicolumn{7}{|c|}{ Indicators } & \multirow{2}{*}{$\begin{array}{l}\text { Key Findings/ } \\
\text { Lessons Learned }\end{array}$} \\
\hline & & & & & & Types of Services & $\begin{array}{l}\text { Program } \\
\text { Duration }\end{array}$ & $\begin{array}{l}\text { Ended/ } \\
\text { Current }\end{array}$ & Types of Training & $\begin{array}{c}\text { Training } \\
\text { Duration } \\
\end{array}$ & Challenges (-) & $\begin{array}{l}\text { Facilitated Factors } \\
\qquad(+)\end{array}$ & \\
\hline 7 & $\begin{array}{l}\text { Peiris et } \\
\text { al. [23] }\end{array}$ & $\begin{array}{c}\text { 2016; } \\
\text { Beijing; } \\
\text { Hebei } \\
\text { Province }\end{array}$ & T2DM & $\begin{array}{c}\text { FHPs; } \\
\text { Healthcare } \\
\text { staff }\end{array}$ & $\begin{array}{l}\text { Healthcare } \\
\text { Staff: Paid; } \\
\text { FHP: unpaid }\end{array}$ & $\begin{array}{l}\text { Healthcare workers: } \\
\text { case monitoring, } \\
\text { provided support to } \\
\text { FHPs via } \\
\text { communication } \\
\text { tools built inside the } \\
\text { mHealth } \\
\text { application; FHPs: } \\
\text { reported the } \\
\text { progress and update } \\
\text { EHR data on behalf } \\
\text { of the patients (i.e., } \\
\text { their family } \\
\text { members who have } \\
\text { diabetes) via the } \\
\text { mHealth } \\
\text { application. } \\
\text { Co-determined } \\
\text { action plan with the } \\
\text { support from } \\
\text { healthcare workers. } \\
\text { Experience sharing } \\
\text { with other FHPs in } \\
\text { the community via } \\
\text { application-based } \\
\text { forum. }\end{array}$ & $\begin{array}{l}2 \text { years: } \\
\text { 2016-2017 }\end{array}$ & $\mathrm{N} / \mathrm{A}$ & $\begin{array}{l}\text { Installation and the } \\
\text { use of the } \\
\text { technology and } \\
\text { management of } \\
\text { diabetes }\end{array}$ & N/A & $\begin{array}{l}\text { Findings were not } \\
\text { generalizable for } \\
\text { other contexts } \\
\text { without EHR } \\
\text { infrastructure, and } \\
\text { for the population } \\
\text { with limited access } \\
\text { to smart-phone } \\
\text { technology }\end{array}$ & $\begin{array}{l}\text { 1. Cost-saving; } \\
\text { 2. time-saving; } \\
\text { 3. strong motivation } \\
\text { of FHPs to support } \\
\text { families with } \\
\text { diabetes; } 4 \text {. close } \\
\text { communication } \\
\text { between clinical } \\
\text { healthcare staffs and } \\
\text { FHPs. }\end{array}$ & $\begin{array}{l}\text { With FHPs' and } \\
\text { community } \\
\text { healthcare staff } \\
\text { engagement, the } \\
\text { application-based } \\
\text { mHealth } \\
\text { intervention had the } \\
\text { potential to increase } \\
\text { the quality of } \\
\text { treatment among } \\
\text { diabetes patients, } \\
\text { and to scale-up the } \\
\text { intervention in } \\
\text { similar settings. }\end{array}$ \\
\hline \multicolumn{14}{|c|}{ Cancer } \\
\hline 8 & $\begin{array}{l}\text { Belinson } \\
\text { et al. [24] }\end{array}$ & $\begin{array}{c}\text { 2014; } \\
\text { Henan } \\
\text { Province }\end{array}$ & $\begin{array}{l}\text { Cervical } \\
\text { Cancer }\end{array}$ & $\begin{array}{l}\text { CLs; } \\
\text { Promoters;local } \\
\text { health } \\
\text { worker }\end{array}$ & al & $\begin{array}{l}\text { Joint tasks for CLs } \\
\text { and promoters: } \\
\text { gathering personal } \\
\text { information and } \\
\text { labeling specimens; } \\
\text { following the } \\
\text { procedures; } \\
\text { promoting screening } \\
\text { via video, posters, } \\
\text { workshops. CLs: } \\
\text { instruct sample } \\
\text { collection. Local } \\
\text { health workers: } \\
\text { consultations and } \\
\text { referrals after results } \\
\text { generation. }\end{array}$ & 2011-2013 & Ended & $\begin{array}{l}\text { Meaning of a } \\
\text { positive test; } \\
\text { management } \\
\text { options and } \\
\text { techniques; via } \\
\text { video and } \\
\text { workshops }\end{array}$ & 1 day & $\mathrm{N} / \mathrm{A}$ & $\begin{array}{l}\text { 1. Good } \\
\text { communication } \\
\text { skills; } 2 \text {. enthusiasm } \\
\text { for the job; } \\
\text { 3. community, } \\
\text { institutional, and } \\
\text { government support }\end{array}$ & $\begin{array}{l}\text { This } \\
\text { community-based } \\
\text { self-sampling model } \\
\text { was capable of } \\
\text { developing massive } \\
\text { screenings. } \\
\text { Improvements can } \\
\text { be made when local } \\
\text { doctors are trained } \\
\text { to manage the } \\
\text { positives. }\end{array}$ \\
\hline
\end{tabular}


Table 1. Cont.

\begin{tabular}{|c|c|c|c|c|c|c|c|c|c|c|c|c|c|}
\hline \multirow{2}{*}{ No. } & \multirow{2}{*}{ Author } & \multirow{2}{*}{$\begin{array}{c}\text { Year; } \\
\text { Location }\end{array}$} & \multirow{2}{*}{$\begin{array}{l}\text { Type of } \\
\text { NCDs }\end{array}$} & \multirow{2}{*}{$\begin{array}{l}\text { Provider } \\
\text { Type }\end{array}$} & \multirow{2}{*}{$\begin{array}{c}\text { Payment } \\
\text { Mechanism/ } \\
\text { Structure } \\
\text { (Paid/Unpaid) }\end{array}$} & \multicolumn{7}{|c|}{ Indicators } & \multirow{2}{*}{$\begin{array}{l}\text { Key Findings/ } \\
\text { Lessons Learned }\end{array}$} \\
\hline & & & & & & Types of Services & $\begin{array}{l}\text { Program } \\
\text { Duration }\end{array}$ & $\begin{array}{l}\text { Ended/ } \\
\text { Current }\end{array}$ & Types of Training & $\begin{array}{c}\text { Training } \\
\text { Duration }\end{array}$ & Challenges (-) & $\begin{array}{l}\text { Facilitated Factors } \\
\qquad(+)\end{array}$ & \\
\hline 9 & $\begin{array}{l}\text { Chai et al. } \\
\text { [14] }\end{array}$ & $\begin{array}{c}\text { 2015; } \\
\text { An'hui } \\
\text { Province }\end{array}$ & cancer & VDs & paid & $\begin{array}{l}\text { 1. Health counseling } \\
\text { regarding alerting } \\
\text { risks and harms, } \\
\text { discussing efficacy } \\
\text { and benefits, and } \\
\text { anticipating barriers; } \\
\text { 2. risk assessment } \\
\text { promotions; } \\
\text { 3. support on } \\
\text { healthy lifestyle } \\
\text { behavioral changes } \\
\text { (reviewing behavior } \\
\text { changes, } \\
\text { encouraging } \\
\text { improvements, } \\
\text { identifying and } \\
\text { solving problems); } \\
\text { 4. manage, record, } \\
\text { and upload typical } \\
\text { cases bi-monthly on } \\
\text { a web forum and } \\
\text { shared experiences } \\
\text { with other experts } \\
\text { and VDs }\end{array}$ & 5 years & On-going & $\begin{array}{l}\text { Web-based tutorial } \\
\text { on implementing } \\
\text { the project } \\
\text { prevention in both } \\
\text { video and textual } \\
\text { formats; typical case } \\
\text { studies as references } \\
\text { for practice; video } \\
\text { and pictorial } \\
\text { materials about } \\
\text { cancer and its } \\
\text { prevention }\end{array}$ & $\begin{array}{l}\text { One-day } \\
\text { orientation } \\
\text { workshop }\end{array}$ & $\begin{array}{l}\text { Heavy reliance on } \\
\text { electronic support, } \\
\text { so the actual } \\
\text { practice may } \\
\text { beyond the ability of } \\
\text { VDs in remote rural } \\
\text { areas to use } \\
\text { computerized } \\
\text { systems }\end{array}$ & $\begin{array}{l}\text { 1. } \\
\text { Performance-based } \\
\text { incentive and } \\
\text { awards; } \\
\text { 2. web-based } \\
\text { support and } \\
\text { supervision system } \\
\text { were time-saving; } \\
\text { 3. user-friendly } \\
\text { education and } \\
\text { learning assistance; } \\
\text { 4. self-practice, } \\
\text { encouragement, } \\
\text { problem inquiry, } \\
\text { and answering in } \\
\text { the training allowed } \\
\text { most village doctors } \\
\text { to become confident } \\
\text { users of the } \\
\text { electronic support } \\
\text { system }\end{array}$ & $\begin{array}{l}\text { This international } \\
\text { program was to } \\
\text { empower advocacy, } \\
\text { raise awareness } \\
\text { about dementia, and } \\
\text { ensure that the } \\
\text { health and social } \\
\text { care needs of older } \\
\text { people were met in } \\
\text { low- and middle- } \\
\text { income countries }\end{array}$ \\
\hline \multicolumn{14}{|c|}{ Mental Health } \\
\hline 10 & $\begin{array}{l}\text { Prince et } \\
\text { al. [25] }\end{array}$ & $\begin{array}{c}\text { 2007; } \\
\text { urban and } \\
\text { rural } \\
\text { catchment, } \\
\text { no specific } \\
\text { location } \\
\text { mentioned }\end{array}$ & dementia & CHWs & paid & $\begin{array}{l}\text { 1. Help researchers } \\
\text { to detect high-risk } \\
\text { populations; } \\
\text { 2. being the } \\
\text { community key } \\
\text { informants of the } \\
\text { research team }\end{array}$ & 2 years & Ended & $\mathrm{N} / \mathrm{A}$ & $\mathrm{N} / \mathrm{A}$ & $\mathrm{N} / \mathrm{A}$ & $\mathrm{N} / \mathrm{A}$ & $\begin{array}{l}\text { Village workers } \\
\text { with non-clinical } \\
\text { training have the } \\
\text { potential to manage } \\
\text { severe psychiatric } \\
\text { diseases and other } \\
\text { chronic conditions. }\end{array}$ \\
\hline
\end{tabular}


Table 1. Cont.

\begin{tabular}{|c|c|c|c|c|c|c|c|c|c|c|c|c|c|}
\hline \multirow{2}{*}{ No. } & \multirow{2}{*}{ Author } & \multirow{2}{*}{$\begin{array}{c}\text { Year; } \\
\text { Location }\end{array}$} & \multirow{2}{*}{$\begin{array}{l}\text { Type of } \\
\text { NCDs }\end{array}$} & \multirow{2}{*}{$\begin{array}{l}\text { Provider } \\
\text { Type }\end{array}$} & \multirow{2}{*}{$\begin{array}{c}\text { Payment } \\
\text { Mechanism/ } \\
\text { Structure } \\
\text { (Paid/Unpaid) } \\
\end{array}$} & \multicolumn{7}{|c|}{ Indicators } & \multirow{2}{*}{$\begin{array}{l}\text { Key Findings/ } \\
\text { Lessons Learned }\end{array}$} \\
\hline & & & & & & Types of Services & $\begin{array}{l}\text { Program } \\
\text { Duration }\end{array}$ & $\begin{array}{l}\text { Ended/ } \\
\text { Current }\end{array}$ & Types of Training & $\begin{array}{c}\text { Training } \\
\text { Duration } \\
\end{array}$ & Challenges (-) & $\begin{array}{l}\text { Facilitated Factors } \\
\qquad(+)\end{array}$ & \\
\hline 11 & $\begin{array}{l}\text { Gong et } \\
\text { al. [26] }\end{array}$ & $\begin{array}{l}\text { 2014; } \\
\text { Liuyang, } \\
\text { Hunan } \\
\text { Province }\end{array}$ & schizophrenia & VDs & paid & $\begin{array}{l}\text { 1. Manage case files } \\
\text { for patients; } 2 \text {. store } \\
\text { and distribute } \\
\text { antipsychotics to } \\
\text { family members on } \\
\text { a weekly basis, or } \\
\text { directly observe } \\
\text { drug-taking at the } \\
\text { village clinic on a } \\
\text { daily basis; } \\
\text { 3. accompany } \\
\text { patients and family } \\
\text { members on } \\
\text { bi-monthly visits to } \\
\text { psychiatrists for } \\
\text { drug dispensation; } \\
\text { 4. record patients' } \\
\text { medication-taking } \\
\text { behavior weekly; } \\
\text { 5. identify signs of } \\
\text { relapse; } 6 \text {. referral. }\end{array}$ & 1 year & Ended & $\begin{array}{l}\text { Mental health } \\
\text { knowledge, } \\
\text { case-management } \\
\text { skills, and DOT. }\end{array}$ & 3 days & $\begin{array}{l}\text { 1. Already overload, } \\
\text { no time for extra } \\
\text { work; 2. no financial } \\
\text { compensation for } \\
\text { extra efforts; } \\
\text { 3. inadequate } \\
\text { engagement from } \\
\text { patients and } \\
\text { patients' families }\end{array}$ & $\begin{array}{l}\text { 1. Under the } \\
\text { national "686" } \\
\text { mental health } \\
\text { scheme-government } \\
\text { support; } \\
\text { 2. consistent } \\
\text { collaboration with } \\
\text { local government; } \\
\text { 3. training met local } \\
\text { VDs' competence } \\
\text { and expectations }\end{array}$ & $\begin{array}{l}\text { The results of this } \\
\text { experiment } \\
\text { provided evidence } \\
\text { on the role of health } \\
\text { workers with } \\
\text { relatively limited } \\
\text { medical training in } \\
\text { managing severe } \\
\text { psychiatric disease } \\
\text { and other chronic } \\
\text { conditions in } \\
\text { developing } \\
\text { countries. }\end{array}$ \\
\hline 12 & $\begin{array}{l}\text { Chen et } \\
\text { al. [27] }\end{array}$ & $\begin{array}{c}\text { 2014; } \\
\text { Xuhui and } \\
\text { Hongkou } \\
\text { Districts; } \\
\text { Shanghai }\end{array}$ & schizophrenia & CHWs & paid & $\begin{array}{l}\text { Worked with } \\
\text { community } \\
\text { psychiatrists and } \\
\text { nurses as a team to } \\
\text { conduct case } \\
\text { management: } \\
\text { 1. Assess recovery } \\
\text { status, employment } \\
\text { status, and social } \\
\text { activities of patients; } \\
\text { 2. assist patients to } \\
\text { develop } \\
\text { personalized } \\
\text { rehabilitation plan } \\
\text { and cope with the } \\
\text { plan, drug } \\
\text { adherence training, } \\
\text { daily skills training, } \\
\text { and family } \\
\text { psychological } \\
\text { intervention; } \\
\text { 3. monthly } \\
\text { follow-up to refine } \\
\text { the plan }\end{array}$ & 2 year & Ended & N/A & N/A & $\mathrm{N} / \mathrm{A}$ & $\mathrm{N} / \mathrm{A}$ & $\begin{array}{l}\text { The study } \\
\text { highlighted the } \\
\text { need to involve } \\
\text { family members in } \\
\text { the management of } \\
\text { patients' } \\
\text { medications, to use } \\
\text { the minimum } \\
\text { effective dosage of } \\
\text { medications, and to } \\
\text { manage all side } \\
\text { effects. }\end{array}$ \\
\hline
\end{tabular}


Table 1. Cont.

\begin{tabular}{|c|c|c|c|c|c|c|c|c|c|c|c|c|c|}
\hline \multirow{2}{*}{ No. } & \multirow{2}{*}{ Author } & \multirow{2}{*}{$\begin{array}{c}\text { Year; } \\
\text { Location }\end{array}$} & \multirow{2}{*}{$\begin{array}{l}\text { Type of } \\
\text { NCDs }\end{array}$} & \multirow{2}{*}{$\begin{array}{l}\text { Provider } \\
\text { Type }\end{array}$} & \multirow{2}{*}{$\begin{array}{c}\text { Payment } \\
\text { Mechanism/ } \\
\text { Structure } \\
\text { (Paid/Unpaid) }\end{array}$} & \multicolumn{7}{|c|}{ Indicators } & \multirow{2}{*}{$\begin{array}{l}\text { Key Findings/ } \\
\text { Lessons Learned }\end{array}$} \\
\hline & & & & & & Types of Services & $\begin{array}{l}\text { Program } \\
\text { Duration }\end{array}$ & $\begin{array}{l}\text { Ended/ } \\
\text { Current }\end{array}$ & Types of Training & $\begin{array}{c}\text { Training } \\
\text { Duration } \\
\end{array}$ & Challenges (-) & $\begin{array}{c}\text { Facilitated Factors } \\
\qquad(+)\end{array}$ & \\
\hline 13 & $\begin{array}{l}\text { Zhou et } \\
\text { al. [28] }\end{array}$ & $\begin{array}{c}\text { 2014; } \\
\text { Shanghai }\end{array}$ & schizophrenia & CHWs & paid & $\begin{array}{l}\text { Assist patients with } \\
\text { self-management. } \\
\text { After each patient } \\
\text { received weekly } \\
\text { self-management } \\
\text { skill training, CHWs } \\
\text { reviewed patients' } \\
\text { self-management } \\
\text { checklist (recorded } \\
\text { their daily } \\
\text { adherence quality of } \\
\text { sleep, occurrence of } \\
\text { side effects, } \\
\text { occurrence of } \\
\text { residual symptoms } \\
\text { and early signs of } \\
\text { relapse, daily } \\
\text { activities, and } \\
\text { general mood) every } \\
\text { month at a group } \\
\text { meeting to } \\
\text { supervise the } \\
\text { adherence and } \\
\text { collect record }\end{array}$ & 2.5 year & Ended & N/A & N/A & N/A & N/A & $\begin{array}{l}\text { Self-management } \\
\text { training could } \\
\text { introduce a } \\
\text { reduction in relapse } \\
\text { and improvements } \\
\text { in chronic } \\
\text { schizophrenia } \\
\text { medication } \\
\text { adherence. } \\
\text { Cost-benefit studies } \\
\text { are needed to assess } \\
\text { the feasibility of } \\
\text { up-scaling this } \\
\text { intervention. }\end{array}$ \\
\hline 14 & $\begin{array}{l}\text { Ma et al. } \\
\text { [29] }\end{array}$ & $\begin{array}{c}\text { 2015; } \\
\text { Guangxi } \\
\text { Province }\end{array}$ & $\begin{array}{l}\text { mental } \\
\text { illness }\end{array}$ & $\begin{array}{l}\text { primary } \\
\text { health care } \\
\text { providers }\end{array}$ & paid & $\begin{array}{l}\text { 1. Community } \\
\text { education; } \\
\text { 2. medication } \\
\text { distribution; } \\
\text { 3. observed } \\
\text { compliance and life } \\
\text { status; } 4 \text {. report side } \\
\text { effects or any } \\
\text { abnormality; } \\
\text { 5. referral and } \\
\text { follow-up }\end{array}$ & 2006-present & Current & $\begin{array}{l}\text { Training provided } \\
\text { by the national ' } 686 \\
\text { project': mental } \\
\text { health disease } \\
\text { management, } \\
\text { education and social } \\
\text { treatment, and } \\
\text { prevention of } \\
\text { mental illnesses }\end{array}$ & N/A & $\begin{array}{l}\text { 1. Lack of } \\
\text { professional } \\
\text { knowledge; } 2 \text {. fear } \\
\text { of patients' attack; } \\
\text { 3. extra workload; } \\
\text { 4. no available } \\
\text { management } \\
\text { approach; } \\
\text { 5. insufficient } \\
\text { subsidies }\end{array}$ & $\begin{array}{l}\text { 1. Communication } \\
\text { skills; 2. proper } \\
\text { attitudes (without } \\
\text { discrimination); } \\
\text { 3. improved } \\
\text { knowledge; } \\
\text { 4. increased } \\
\text { income/subsidy }\end{array}$ & $\begin{array}{l}\text { Improvements can } \\
\text { be made regarding } \\
\text { 1. trainings on } \\
\text { professional mental } \\
\text { health knowledge } \\
\text { and attitudes; } \\
\text { 2. management } \\
\text { approach; } \\
\text { 3. income/subsidy }\end{array}$ \\
\hline
\end{tabular}


Table 1. Cont.

\begin{tabular}{|c|c|c|c|c|c|c|c|c|c|c|c|c|c|}
\hline \multirow{2}{*}{ No. } & \multirow{2}{*}{ Author } & \multirow{2}{*}{$\begin{array}{c}\text { Year; } \\
\text { Location }\end{array}$} & \multirow{2}{*}{$\begin{array}{l}\text { Type of } \\
\text { NCDs }\end{array}$} & \multirow{2}{*}{$\begin{array}{l}\text { Provider } \\
\text { Type }\end{array}$} & \multirow{2}{*}{$\begin{array}{l}\text { Payment } \\
\text { Mechanism/ } \\
\text { Structure } \\
\text { (Paid/Unpaid) }\end{array}$} & \multicolumn{7}{|c|}{ Indicators } & \multirow{2}{*}{$\begin{array}{l}\text { Key Findings/ } \\
\text { Lessons Learned }\end{array}$} \\
\hline & & & & & & Types of Services & $\begin{array}{l}\text { Program } \\
\text { Duration }\end{array}$ & $\begin{array}{l}\text { Ended/ } \\
\text { Current }\end{array}$ & Types of Training & $\begin{array}{l}\text { Training } \\
\text { Duration }\end{array}$ & Challenges (-) & $\begin{array}{l}\text { Facilitated Factors } \\
\qquad(+)\end{array}$ & \\
\hline 15 & $\begin{array}{l}\text { Tang et } \\
\text { al. [30] }\end{array}$ & $\begin{array}{c}\text { 2015; } \\
\text { Mianzhu, } \\
\text { Sichuann } \\
\text { province }\end{array}$ & $\begin{array}{c}\text { late-life } \\
\text { depression }\end{array}$ & VDs & not paid & $\begin{array}{l}\text { Conducted weekly } \\
\text { intervention with } \\
\text { elderly depression } \\
\text { patients using CBT } \\
\text { techniques to } \\
\text { 1. perform physical } \\
\text { examinations; } \\
\text { 2. identify emotion } \\
\text { status and negative } \\
\text { thoughts; } 3 \text {. proceed } \\
\text { psychological } \\
\text { interventions; } \\
\text { 4. provide problem } \\
\text { solving methods }\end{array}$ & 2 months & Current & $\begin{array}{l}\text { Workshops on } \\
\text { mental disorder } \\
\text { knowledge, } \\
\text { counseling concepts, } \\
\text { and techniques, } \\
\text { with specific focus } \\
\text { on CBT. Practice } \\
\text { through role-play. } \\
\text { Trainings were } \\
\text { conducted by a } \\
\text { qualified cognitive } \\
\text { therapist }\end{array}$ & $\begin{array}{l}\text { Six full days } \\
\text { (three } \\
\text { consecutive } \\
\text { weekends) }\end{array}$ & $\begin{array}{l}\text { 1. Time constraint } \\
\text { for training; } \\
\text { 2. under-developed } \\
\text { training manuals } \\
\text { and inadequate } \\
\text { practice 3. poor } \\
\text { patient } \\
\text { adherence-preferred } \\
\text { medicine over CBT; } \\
\text { 4. no financial } \\
\text { incentive }\end{array}$ & $\begin{array}{l}\text { 1. Well designed } \\
\text { (easy to understand } \\
\text { the content) and } \\
\text { organized (the use } \\
\text { of role play) } \\
\text { training; } 2 \text {. strong } \\
\text { learning ability and } \\
\text { interest; } 3 \text {. local } \\
\text { community trust; } \\
\text { 4. multi-disciplinary } \\
\text { team }\end{array}$ & $\begin{array}{l}\text { The study } \\
\text { highlighted the } \\
\text { feasibility and good } \\
\text { patient acceptability } \\
\text { of including CBT in } \\
\text { the treatment } \\
\text { process for the rural } \\
\text { elderly. Remaining } \\
\text { challenges: } 1 \text {. A lack } \\
\text { of step-by-step } \\
\text { treatment manuals } \\
\text { in the training; } 2 \text {. a } \\
\text { lack of support from } \\
\text { family; } 3 \text {. a need to } \\
\text { integrate CBT } \\
\text { intervention into } \\
\text { public health } \\
\text { services. }\end{array}$ \\
\hline 16 & $\begin{array}{l}\text { Xu et al. } \\
\text { [31] }\end{array}$ & $\begin{array}{c}\text { 2016; } \\
\text { Liuyang, } \\
\text { Hunan } \\
\text { Province }\end{array}$ & schizophrenia & $\begin{array}{l}\text { VDs; } \\
\text { LHS: } \\
\text { mostly } \\
\text { family } \\
\text { members } \\
\text { of the } \\
\text { patients }\end{array}$ & $\begin{array}{l}\text { VD: paid; } \\
\text { LHS: unpaid }\end{array}$ & $\begin{array}{l}\text { VDs: } 1 \text {. Screening, } \\
\text { as the "686" scheme } \\
\text { requires; } 2 \text {. reported } \\
\text { relapse signs and } \\
\text { side effects to } \\
\text { psychiatrists; } \\
\text { 3. teamed up with } \\
\text { LHSs, MHA, and } \\
\text { psychiatrists to } \\
\text { assist urgent care. } \\
\text { LHSs: } 1 \text {. Facilitated } \\
\text { patient medication } \\
\text { adherence with } \\
\text { prompts from the } \\
\text { e-reminders; } \\
\text { 2. monitored for } \\
\text { early signs of } \\
\text { relapse and side } \\
\text { effects using } \\
\text { checklists from the } \\
\text { e-monitor and } \\
\text { report to VDs; } \\
\text { 3. teamed up with } \\
\text { the VDs and the } \\
\text { township MHA to } \\
\text { facilitate treatment } \\
\text { adjustments and } \\
\text { urgent care }\end{array}$ & 1 year & On-going & $\begin{array}{l}\text { The built-in } \\
\text { e-educator mHealth } \\
\text { program sent } \\
\text { periodic SMS } \\
\text { messages to } \\
\text { patients, LHSs, } \\
\text { MHA, and VDs to } \\
\text { educate them on } \\
\text { schizophrenia } \\
\text { symptoms, } \\
\text { medication, } \\
\text { adherence strategies, } \\
\text { relapse, } \\
\text { rehabilitation, and } \\
\text { social resources }\end{array}$ & N/A & $\begin{array}{l}\text { 1. Local } \\
\text { psychiatrists with } \\
\text { limited training may } \\
\text { deliver } \\
\text { inappropriate } \\
\text { services; 2. funding } \\
\text { for the program may } \\
\text { not be sustainable in } \\
\text { the future. }\end{array}$ & $\begin{array}{l}\text { 1. Policy support; } \\
\text { 2. individual and } \\
\text { community } \\
\text { engagement (MHAs, } \\
\text { psychiatrists, VDs, } \\
\text { patients and their } \\
\text { families); VDs: No } \\
\text { additional } \\
\text { workload; LHSs: } \\
\text { 1. Care and love for } \\
\text { their families (i.e., } \\
\text { patients) as the } \\
\text { major job } \\
\text { motivation; } \\
\text { 2. non-monetary } \\
\text { reward. }\end{array}$ & $\begin{array}{l}\text { The design of the } \\
\text { LEAN program was } \\
\text { expected to be a } \\
\text { cost-saving, feasible, } \\
\text { and generalizable } \\
\text { community-based } \\
\text { schizophrenia } \\
\text { management model } \\
\text { to improve } \\
\text { medication } \\
\text { adherence in } \\
\text { comparable } \\
\text { socio-economic } \\
\text { contexts where } \\
\text { humanand financial } \\
\text { resources were } \\
\text { limited. Long-term } \\
\text { cost-effectiveness } \\
\text { assessment is } \\
\text { needed. }\end{array}$ \\
\hline
\end{tabular}


Table 1. Cont.

\begin{tabular}{|c|c|c|c|c|c|c|c|c|c|c|c|c|c|}
\hline \multirow{2}{*}{ No. } & \multirow{2}{*}{ Author } & \multirow{2}{*}{$\begin{array}{c}\text { Year; } \\
\text { Location }\end{array}$} & \multirow{2}{*}{$\begin{array}{l}\text { Type of } \\
\text { NCDs }\end{array}$} & \multirow{2}{*}{$\begin{array}{c}\text { Provider } \\
\text { Type }\end{array}$} & \multirow{2}{*}{$\begin{array}{c}\text { Payment } \\
\text { Mechanism/ } \\
\text { Structure } \\
\text { (Paid/Unpaid) }\end{array}$} & \multicolumn{7}{|c|}{ Indicators } & \multirow{2}{*}{$\begin{array}{l}\text { Key Findings/ } \\
\text { Lessons Learned }\end{array}$} \\
\hline & & & & & & Types of Services & $\begin{array}{l}\text { Program } \\
\text { Duration }\end{array}$ & $\begin{array}{l}\text { Ended/ } \\
\text { Current }\end{array}$ & Types of Training & $\begin{array}{c}\text { Training } \\
\text { Duration }\end{array}$ & Challenges (-) & $\begin{array}{l}\text { Facilitated Factors } \\
\qquad(+)\end{array}$ & \\
\hline \multicolumn{14}{|c|}{ Cardiovascular diseases } \\
\hline 17 & $\begin{array}{l}\text { Ajay et al. } \\
\text { [32] }\end{array}$ & $\begin{array}{l}2014 ; \\
\text { Gongbujiang-da } \\
\text { county, } \\
\text { Linzhou } \\
\text { county, } \\
\text { Tibet } \\
\text { Province }\end{array}$ & CVD & $\begin{array}{l}\text { Community } \\
\text { health } \\
\text { workers(CHWs) }\end{array}$ & paid & $\begin{array}{l}\text { With the } \\
\text { smartphone-based } \\
\text { electronic decision } \\
\text { support, CHWs can } \\
\text { provide 1. monthly } \\
\text { follow-up care; } \\
\text { 2. high-risk people } \\
\text { identification; } \\
\text { 3. referral; } \\
\text { 4. therapeutic } \\
\text { lifestyle advice } \\
\text { (smoking cessation } \\
\text { and salt reduction); } \\
\text { 5. prescribe two } \\
\text { drugs (blood } \\
\text { pressure lowering } \\
\text { drugs and aspirin) }\end{array}$ & 1 year & Ended & $\begin{array}{l}\text { Training on the } \\
\text { intervention } \\
\text { protocol, including } \\
\text { key messages on } \\
\text { targeted CVD } \\
\text { lifestyle risks and } \\
\text { medications }\end{array}$ & $\begin{array}{l}\text { Initial } \\
\text { training: not } \\
\text { mentioned; } \\
\text { refresh } \\
\text { training: 1-3 } \\
\text { months after } \\
\text { intervention } \\
\text { began }\end{array}$ & $\begin{array}{l}\text { Lack of economic } \\
\text { and healthcare } \\
\text { resources }\end{array}$ & $\begin{array}{l}\text { Supportive national } \\
\text { guidelines and } \\
\text { policies on CVD } \\
\text { prevention and } \\
\text { control }\end{array}$ & $\begin{array}{l}\text { The first study to } \\
\text { evaluate the effects } \\
\text { of a simplified } \\
\text { management } \\
\text { program delivered } \\
\text { by CHWs with the } \\
\text { help of electronic } \\
\text { decision support } \\
\text { system on } \\
\text { improving the } \\
\text { health of high CVD } \\
\text { risk patients. If } \\
\text { effective, this } \\
\text { intervention } \\
\text { strategy can serve as } \\
\text { a generalized model } \\
\text { for similar settings }\end{array}$ \\
\hline 18 & $\begin{array}{l}\text { Yan et al. } \\
\text { [33] }\end{array}$ & $\begin{array}{l}\text { 2014; } \\
\text { Hebei, } \\
\text { Liaoning, } \\
\text { Ningxia, } \\
\text { Shanxi and } \\
\text { Shaanxi }\end{array}$ & CVD & $\begin{array}{l}\text { Village } \\
\text { Doctors }\end{array}$ & paid & $\begin{array}{l}\text { 1. Identified } \\
\text { high-risk } \\
\text { individuals by } \\
\text { screening all } \\
\text { patients in the } \\
\text { village clinics. } \\
\text { 2. contacted patients } \\
\text { with existing } \\
\text { diseases or } \\
\text { potentially at high } \\
\text { risk to maximize } \\
\text { screening; } \\
\text { 3. measured blood } \\
\text { pressure; } \\
\text { 4. provided lifestyle } \\
\text { modification advice; } \\
\text { 5. monitored acute } \\
\text { symptoms or early } \\
\text { signs of clinical } \\
\text { events on monthly } \\
\text { follow-up; } \\
\text { 4) referral }\end{array}$ & 2 years & Ended & $\begin{array}{l}\text { A technical package } \\
\text { developed to guide } \\
\text { VDs on how to } \\
\text { screen, identify, } \\
\text { treat, follow-up, and } \\
\text { refer cardiovascular } \\
\text { high-risk } \\
\text { individuals during } \\
\text { their routine } \\
\text { services. }\end{array}$ & $\begin{array}{l}\text { Two 1-day } \\
\text { sessions:1 } \\
\text { before } \\
\text { intervention, } \\
\text { and again 1 } \\
\text { month after } \\
\text { the initiation } \\
\text { of } \\
\text { intervention }\end{array}$ & $\mathrm{N} / \mathrm{A}$ & $\begin{array}{l}\text { 1. Performance- } \\
\text { based feedbacks and } \\
\text { financial } \\
\text { incentivespayment } \\
\text { increased } \\
\text { motivation; } \\
\text { 2. interventions } \\
\text { were designed to fit } \\
\text { CVD management } \\
\text { in resource-limited } \\
\text { areas }\end{array}$ & $\begin{array}{l}\text { This was the first } \\
\text { cluster-randomized } \\
\text { trial in the world to } \\
\text { assess the } \\
\text { population impacts } \\
\text { of a high-risk } \\
\text { strategy in } \\
\text { prevention and } \\
\text { control of CVD. The } \\
\text { technical } \\
\text { interventions used } \\
\text { were all } \\
\text { evidence-based and } \\
\text { tailored for VDs. }\end{array}$ \\
\hline
\end{tabular}


Table 1. Cont.

\begin{tabular}{|c|c|c|c|c|c|c|c|c|c|c|c|c|c|}
\hline \multirow{2}{*}{ No. } & \multirow{2}{*}{ Author } & \multirow{2}{*}{$\begin{array}{c}\text { Year; } \\
\text { Location }\end{array}$} & \multirow{2}{*}{$\begin{array}{l}\text { Type of } \\
\text { NCDs }\end{array}$} & \multirow{2}{*}{$\begin{array}{l}\text { Provider } \\
\text { Type }\end{array}$} & \multirow{2}{*}{$\begin{array}{c}\text { Payment } \\
\text { Mechanism/ } \\
\text { Structure } \\
\text { (Paid/Unpaid) }\end{array}$} & \multicolumn{7}{|c|}{ Indicators } & \multirow{2}{*}{$\begin{array}{l}\text { Key Findings/ } \\
\text { Lessons Learned }\end{array}$} \\
\hline & & & & & & Types of Services & $\begin{array}{l}\text { Program } \\
\text { Duration }\end{array}$ & $\begin{array}{l}\text { Ended/ } \\
\text { Current }\end{array}$ & Types of Training & $\begin{array}{l}\text { Training } \\
\text { Duration }\end{array}$ & Challenges (-) & $\begin{array}{l}\text { Facilitated Factors } \\
\qquad(+)\end{array}$ & \\
\hline 19 & $\begin{array}{l}\text { Tian et al. } \\
\text { [15] }\end{array}$ & $\begin{array}{l}\text { 2015; } \\
\text { Gongbu- } \\
\text { jiangda } \\
\text { county, } \\
\text { Linzhou } \\
\text { county, } \\
\text { Tibet } \\
\text { Province }\end{array}$ & $\begin{array}{l}\text { cardiovascular } \\
\text { disease } \\
\text { (CVD) }\end{array}$ & $\begin{array}{l}\text { Community } \\
\text { health } \\
\text { workers } \\
\text { (CHWs) }\end{array}$ & paid & $\begin{array}{l}\text { With the } \\
\text { smartphone-based } \\
\text { electronic decision } \\
\text { support, CHWs } \\
\text { provided 1. monthly } \\
\text { follow-up care; } \\
\text { 2. high-risk-patient } \\
\text { identification; } \\
\text { 3. referral; } \\
\text { 4. therapeutic } \\
\text { lifestyle advice } \\
\text { (smoking cessation } \\
\text { and salt reduction); } \\
\text { prescription of two } \\
\text { drugs (blood } \\
\text { pressure lowering } \\
\text { drugs and Aspirin); } \\
\text { 5. screening for new } \\
\text { symptoms, diseases, } \\
\text { and side effects; } 6 . \\
\text { blood pressure } \\
\text { measurement }\end{array}$ & 1 year & Ended & $\begin{array}{l}\text { Training on the } \\
\text { intervention } \\
\text { protocol, including } \\
\text { key knowledge and } \\
\text { skill on targeted } \\
\text { CVD lifestyle risk } \\
\text { factors and } \\
\text { medications. }\end{array}$ & $\begin{array}{l}\text { Initial } \\
\text { training: } \\
\text { duration not } \\
\text { mentioned; } \\
\text { refresher } \\
\text { training: } 1-4 \\
\text { months after } \\
\text { the } \\
\text { intervention } \\
\text { began }\end{array}$ & $\begin{array}{l}\text { 1. The duration of } \\
\text { the intervention was } \\
\text { too short to observe } \\
\text { significant health } \\
\text { behavioral change; } \\
\text { 2. lack of resources } \\
\text { in remote areas }\end{array}$ & $\begin{array}{l}\text { 1. Performance- } \\
\text { based incentives. }\end{array}$ & $\begin{array}{l}\text { The CHWs were } \\
\text { capable of } \\
\text { delivering CVD } \\
\text { management } \\
\text { program with } \\
\text { smartphone-based } \\
\text { decision support } \\
\text { system in rural } \\
\text { settings in China } \\
\text { and India. }\end{array}$ \\
\hline \multicolumn{14}{|c|}{ NCD risk factors } \\
\hline 20 & $\begin{array}{l}\text { Li et al. } \\
\text { [34] }\end{array}$ & $\begin{array}{l}\text { 2016; } \\
\text { Hebei, } \\
\text { Liaoning, } \\
\text { Shanxi and } \\
\text { Shaanxi } \\
\text { provinces } \\
\text { and the } \\
\text { Ningxia } \\
\text { Autonomous } \\
\text { Region }\end{array}$ & $\begin{array}{l}\text { sodium } \\
\text { reduction }\end{array}$ & $\begin{array}{l}\text { Village } \\
\text { doctor }\end{array}$ & paid & $\begin{array}{l}\text { Worked with } \\
\text { township health } \\
\text { educators to } 1 . \\
\text { provide health } \\
\text { education in forms } \\
\text { of public lectures; } \\
\text { 2. distribute } \\
\text { promotional } \\
\text { materials; } 3 \text {. conduct } \\
\text { education sessions } \\
\text { with vascular } \\
\text { high-risk } \\
\text { population; } \\
\text { 4. promote salt } \\
\text { substitute }\end{array}$ & 18 months & Ended & $\mathrm{N} / \mathrm{A}$ & $\mathrm{N} / \mathrm{A}$ & $\mathrm{N} / \mathrm{A}$ & $\mathrm{N} / \mathrm{A}$ & $\begin{array}{l}\text { Population sodium } \\
\text { intake was reduced } \\
\text { by this intervention, } \\
\text { through increased } \\
\text { use of salt substitute. } \\
\text { Larger effects could } \\
\text { be achieved in rural } \\
\text { China by a } \\
\text { wholesale switch } \\
\text { from salt to salt } \\
\text { substitute to prevent } \\
\text { stroke. }\end{array}$ \\
\hline
\end{tabular}

Annotations: N/A, no relevant information in the article. Abbreviations: BPHS, Basic Public Health Service; CBT, cognitive behavioral therapy; CHSC, Community Health Service Center; CHS, Community Health Station; CHWs, community health workers; CLs, community leaders; CVD, cardiovascular disease; EHR, electronic health record; FHPs, family health promoters; LEAN, lay health supporter, e-platform, award, and integration; LHSs, lay health supporters; mHealth, mobile health; NCDs, non-communicable diseases; NCMS, New Cooperative Medical Scheme; PHC, primary health care; PLs, peer leaders; PLSP, Peer Leader Support Program; T2DM, type-2 diabetes; VDs, village doctors; DOT, directly observed therapy; SMS, short messaging service; MHA, mental health assistant. 


\section{Results}

\subsection{General Description}

The literature search yielded 396 potentially relevant articles. After screening for eligibility and reviewing inclusion and exclusion criteria, twenty studies were retained (Figure 1). As shown in Table 1 , of these twenty studies [13-15,18-33], eleven studies were single-site programs [13,14,18-20,24-26,28-31]; nine studies discussed multi-site programs [15,21-23,27,31-34]; while two of them did not mention specific locations of the study sites [22,25]. Among the programs with specific study sites $(n=18)$, the majority were located in the east and central parts of China $(n=11)[14,18,20,21,23-28,31]$, followed by western $(n=5)$ [15,30,32-34], northern $(n=1)$ [19], and southern China $(n=1)$ [29].

Most programs included in this review lasted over one year $[13,14,18,19,23-29]$, while a few lasted five years or more [14,18,29]. Five studies were on-going programs [13,14,18,29,31].

The title of the CHWs varied in different programs. Ten programs named the CHWs as 'village doctors' [14,18-20,22,26,30,31,33,34], while the other ten programs [13,15,21,23-25,27-29,32] used 'health coach', 'local health workers', 'CHWs', 'lay family health promoters', 'community health service center staff', and 'primary health care providers'.

\subsection{NCD-Related Services Provided by CHWs}

NCD-services provided by CHWs are categorized in five groups: (1) diabetes and/or hypertension (seven studies) [13,18-23]; (2) cancer (two studies) [14,24]; (3) mental health (seven studies) [25-31]; (4) CVDs (three studies) [15,32,33]; and (5) NCD risk factors (one study) [34].

\subsubsection{Diabetes and Hypertension}

All seven papers that focused on diabetes and hypertension, case management, prevention, and monitoring were the major responsibilities of CHWs. Outreach services such as follow-up via home visits [13,18-21] and behavioral change counseling on healthy diet, physical activities, mental health, and high-risk behavior prevention $[13,18,20,21]$ were largely provided by CHWs in these studies. In two studies, CHWs promoted and conducted screening, or assisted in early detection of high-risk populations $[18,20]$.

\subsubsection{Cancer}

In the program developed by Belinson et al. [24] for community-based cervical cancer screening, community leaders and CHWs worked together to help health professionals gather personal information and promoted the program via video, posters, and educational workshops within the communities. Another on-going study conducted by Chai et al. [14] introduced a systematic approach in cancer prevention by engaging village doctors.

\subsubsection{Mental Health}

Among the seven studies under the mental health category, four focused on schizophrenia [26-28,31], one on dementia [25], one on late-life depression [30], and one on general mental illness [29].

\subsubsection{Cardiovascular Diseases (CVDs)}

The CHWs in all the three CVDs programs [15,32,33] shared similar responsibilities: identifying high-risk patients, providing lifestyle modification recommendations, measuring blood pressure, and referring patients to higher-level clinics. 


\subsubsection{NCD Risk Factors}

Only one study described the NCD risk factors [34]. In this large-scale salt reduction intervention, village doctors distributed promotional materials and salt substitutes, and conducted interactive education for people with a high risk of vascular diseases.

\subsection{Training Received by CHWs}

Fourteen studies had at least some description of the training content received by CHWs [13,15,18,20,22-24,26,29-33], among which eight studies [13-15,24,26,30,32,33] included detailed information about the training procedures and training duration. Training contents reported was program-specific $[15,32,33]$, while training types included web-based training $[14,18,20,31]$ and workshops $[13,24,30]$.

\subsection{Barriers}

Thirteen studies discussed different barriers of engaging CHWs in NCD prevention and control programs [13-15,18-23,26,29-32], as summarized below:

\subsubsection{Lack of Support from the Local Health Systems and from the Public}

Inadequate support from the health system and policy was one of the major barriers in four articles $[19,22,26,29]$. Particularly, Li et al. [22] stated that some rural villages were not covered by the New Cooperative Medical Scheme; therefore, the local CHWs did not have official contracts, which caused dissatisfaction among the CHWs. A lack of support from patients also reduced $\mathrm{CHWs}^{\prime}$ job motivations $[20,26,29,30]$.

\subsubsection{Lack of Resources}

Ajay et al. [32] and Tian et al. [15] pointed out that limited economic and health care resources in the poor areas were the major barriers. $\mathrm{Xu}$ et al. [31] reported that inadequate local psychiatrists compromised the village doctors' performance, as they did not have professional back-up to manage difficult cases.

\subsubsection{Heavy Workload}

Three papers reported that heavy workload with the already assigned services was a barrier for CHWs to provide NCD-related services $[18,26,29]$. The village doctors who were already busy as general primary health care providers were reluctant to increase their workload by adding extra NCD tasks $[18,26,29]$.

\subsubsection{Inadequate Financial Incentives}

In three programs, CHWs were not fully satisfied with the financial incentives they had been receiving $[22,29,30]$. In these studies, their current wage was lower than their expected wages.

\subsubsection{Heavy Reliance on Electronic Technology}

Several studies discussed problems associated with the increasing reliance on technology in the intervention delivery by CHWs [14,18,20,23]. First, Chen et al. [20] and Peiris et al. [23] claimed that even smartphones service was still not accessible for regions with extremely poor conditions. Second, Feng et al. [18] and Chai et al. [14] indicated that the computerized system operation might go beyond the capability of village doctors in remote areas. 


\subsubsection{Lack of Knowledge and Skills}

Three studies reported the inadequate knowledge and skills of CHWs as a barrier $[18,29,30]$. In a CHW-led diabetes prevention program conducted by Feng et al. [18], most village doctors were unaware of the importance of diabetes prevention. In another paper by Ma et al. [29], CHWs' lack of professional mental health expertise limited their capabilities of providing relevant services. One study [30] revealed the unsatisfying training quality by highlighting the limited time for practice.

\subsection{Facilitators}

Sixteen studies [13-15,18-24,26,29-33] indicated at least one facilitator for CHWs to provide NCD prevention and control services. The following categories present a summary of the enabling factors identified:

\subsubsection{Integrated Health System}

Nine studies mentioned that a close collaboration among policy makers, high-level public health institutes, and CHWs was beneficial [18,19,22-24,26,30-32]. In Feng et al. [18], the village clinics prepared appropriate settings and resources to make it convenient for $\mathrm{CHWs}$ to measure blood pressure and conduct counseling. In another diabetes management program [22], village doctors were better managed when they had access to contracts to the New Cooperative Medical Scheme.

\subsubsection{Community and Patient Trust}

Trust from patients and communities was another facilitating factor [12,16,18,26,27]. Particularly in the studies of Feng et al. [18] and Xu et al. [31], mutual trust between CHWs and patients facilitated smooth delivery of CHWs' work, and lead to better medication adherence and patients' satisfaction.

\subsubsection{Incentives}

Although no study mentioned that monetary incentives could engage CHWs in the delivery of NCD programs, the village doctors in one hypertension and diabetes control program confessed that they hoped to receive additional subsidy [22]. Moreover, performance-based incentives were considered efficient in increasing $\mathrm{CHWs}$ job motivations and improving their work performance $[14,15,18,33]$.

\subsubsection{Appropriate Training}

In multiple NCD programs, the trainings were designed to meet $\mathrm{CHWs}^{\prime}$ competences and expectations, so that CHWs were able to quickly understand key concepts and acquire skills $[15,18,22,26,30]$.

\subsubsection{Capacity of CHWs}

Five articles $[13,21,24,29,30]$ included $\mathrm{CHW}$ s $^{\prime}$ capacity (i.e., competencies and communication skills) to deliver the required services as a positive influencing factor. Browning et al. [13] observed that CHWs with better learning capacity could quickly transform the knowledge and skills into practice. Belinson et al. [24] and Ma et al. [29] found that CHWs who possessed high communication skills could effectively assist patients in changing unhealthy behaviors.

\subsubsection{Interests and Attitudes}

Three studies discussed CHW's interests and attitudes as facilitators. Belinson et al. [24] and Tang et al. [30] stated that CHWs were strongly enthusiastic about providing related services in their studies on promoting cervical cancer screening and conducting cognitive behavioral therapy intervention. Another study about mental health care conducted by Ma et al. [29] reported that CHWs 
without discrimination towards mental illness could more effectively communicate with patients and provide appropriate care.

\section{Discussion}

This review provided contemporary evidence of the roles that CHWs could serve in preventing and controlling NCDs in China, and the corresponding barriers and enablers. Despite variations in the study settings, methods, and program durations of the identified papers in this review, the findings indicated that Chinese $\mathrm{CHWs}$ provided certain types of community-based preventive services on major chronic diseases. These were generally aligned with the results of other international reviews [35-38]. However, limited studies in this review focused on NCD risk factors management among general populations, except one sodium reduction program conducted by Li et al. [34]. Common preventable NCD risk factors should not be neglected in community-based NCD programs [39]. Studies in other low- and middle-income countries (LMICs) introduced $\mathrm{CHWs}^{\prime}$ roles in promoting tobacco control, physical activities, and healthy diets among non-patients [40-43]. One program conducted by Reininger et al. [41] integrated CHWs' home visits with the community campaign to educate the Mexican descent population about the significance of physical activity and healthy food consumption in reducing NCDs. The results showed that the participants who received both CHW meetings and radio messages were likely to increase physical activity, and the participants who were exposed to the program were likely to consume more fruit and vegetables than those who were not. These studies underscore the potential to engage CHWs in the delivery of NCDs risk factor reduction intervention; however, further studies on CHWs' feasibility in providing such services in China are needed.

The barriers that we identified in the current study (i.e., inadequate knowledge and skills; heavy workload; limited resources and support) are consistent with the findings in other local and international studies [22,37,44,45]. Consistent with other studies [46-50], the insufficient knowledge and skills of CHWs, as was identified in the current study, could possibly be resolved by delivering organized training that will strengthen CHW's skills and promote positive attitudes towards NCD care at the community level.

The lack of support from the health system was identified as a key challenge in this review. While evidence from the identified articles [18,19,22-24,26,30-32] and previous studies [51-53] also indicated that the support from integrated management and local collaboration would lead to effective CHW-led interventions and assist in scaling-up programs. The integrated management strategy has been enacted since the 2009 health sector reform in the rural health care service scheme. It requires local township health centers (THCs) to take charge of village clinic management, including personnel, finance, facilities, medicine, and all works associated with village doctors [53]. Rural CHWs can seek training, as well as financial and facility support through the contracts with THCs [54]. However, limited to local economic and government interests, not all the CHWs can acquire the support they need.

One unexpected finding in this review was that only three identified papers listed low individual financial incentives as a barrier, while other domestic and international studies included it as a major challenge for $\mathrm{CHWs}^{\prime}$ engagement $[22,45,55,56]$. Chinese CHWs are paid by the central and local governments [52,53], thus it seems that they have relatively less economic difficulties compared with the voluntary or informally-paid CHWs in other countries. However, in fact, researchers have revealed that multiple income disparities exist among CHWs in China. First, although the government pays one part of the CHWs subsidy based on national standard, the delivery of the other parts of the subsidy managed by local THCs vary largely in different settings and are subjected to the local fiscal ability [57]. In addition, despite that local THCs should pay no less than $40 \%$ of the government funding to the village clinics, the THC supervisors sometimes delayed or withheld the funds as a punishment strategy for those village doctors who did not accomplish all the assigned tasks [58]. Furthermore, the New Cooperative Medical Scheme (NCMS) covers healthcare at THCs and county-level hospitals, while it does not cover all village clinics. Patients covered by NCMS would prefer going to the THCs and county-level hospitals rather than the village clinics [59]. As a result, the village doctors would lose 
the opportunities to both receive subsidy from the policy and provide needed care to the patients. The findings in this review $[14,15,18,33]$ suggested that performance-based incentives were widely used as an income strategy to motivate and engage CHWs in the delivery of NCD-related health interventions, although the large-scale validity is uncertain. Therefore, future studies and policies need to be concerned about how to expand the coverage of NCMS contract to all village clinics, how to allocate funding and limited resources to better remunerate and motivate $\mathrm{CHWs}$, and how to ensure commitments to human resources development and capacity building [6,60-62].

We found that several factors (i.e., receiving training, competencies, and skills to deliver NCD-related services, as well as community trust) encouraged CHWs to engage in the delivery of NCD-related services. While we argue that community trust towards CHWs could be affected by the local culture in receiving healthcare services from non-medical professionals, and whether CHWs are well known within the community, measures need to be taken to improve CHWs' capacity as well as trust within the community.

There are several limitations of this study. First, this review excluded many studies considering their absence of information on $\mathrm{CHWs}^{\prime}$ training, services provided, and/or influencing factors that could not meet our inclusion criteria. This information shortage itself could indicate a gap in the current research field that Chinese $\mathrm{CHWs}^{\prime}$ roles as formal ordinary healthcare providers in NCD prevention and control are still neglected, thus further studies to comprehensively explore their potential capability in NCD care provision are needed. Second, this review did not focus on effectiveness of the selected studies. As the aim of the study is to explain the current role and the feasibility of Chinese CHWs, whether those programs including CHWs would have an efficient outcome was not further explored. Therefore, we included some on-going programs that did not offer any evaluation. Further follow-up investigations on the evaluation of those on-going studies are needed in order to assess the contribution of getting CHWs involved in these programs. Third, despite that most identified studies in this review showed that involving CHWs in the programs would likely generate positive program outcomes, there were limitations on the generalizability of those findings. For instance, the program successfully conducted in the urban sites would not be replicable for the population in rural settings because of socio-cultural differences, socio-economic constraints, and study design variations. Also, a comprehensive cost-effectiveness analysis was not mentioned or planned in any of the identified studies. The measures and approaches taken for intervention effectiveness evaluation varied greatly across programs. Future studies with rigorous study designs among larger populations are needed to clarify the generalizability of the findings and to establish more appropriate models in planning, implementing, and evaluating large-scale CHW-led NCD intervention programs. Also, applicability of such programs in both rural and urban capabilities and expectations would be needed. Systematic efforts from and close communication between the central and local stakeholders are warranted during the entire process. Finally, as a result of resource constraints, this review only included English-written studies. In the next stage, a systematic review of the Chinese literature would be integrated as one critical supplementary part to the current study.

\section{Conclusions}

Overall, this review has provided insights into the pattern of NCD-related health interventions that are provided by CHWs in China, and summarized potential barriers and facilitating factors. The findings suggest that Chinese CHWs, upon receiving training, are capable of delivering non-invasive health care services for patients with chronic diseases; however, $\mathrm{CHWs}$ ' engagement in the delivery of NCD risk factor reduction interventions for general population was not common. The barriers and facilitators identified ranged from personal level to health system level factors that could influence $\mathrm{CHWs}^{\prime}$ working motivation and performance. These factors should be taken into account by policy makers and other stakeholders to develop strategies for effectively using the workforce of CHWs in the grass-root NCD prevention and control program implementations. 
Author Contributions: A.S.A. planned the study and oversaw the overall review process. H.L. and W.H. conducted the reviews, collected review articles, and summarized the findings. J.L. and S.T. (Sha Tao) helped to update the review papers and commented on the final draft. P.Z. and S.T. (Shenglan Tang) critically reviewed the first draft of the manuscript and commented on the final draft. H.L. prepared the first draft, which was then distributed to all the co-authors for comments. All authors approved the final draft of the paper.

Funding: Asia Pacific Observatory on Health Systems and Policies of the World Health Organization (Purchase Order 201710952; PI: Abu Abdullah).

Acknowledgments: This paper forms part of the outputs emanating from the Research Hub of Asia Pacific Observatory on Health Systems and Policies (APO) hosted by the Global Health Research Center of Duke Kunshan University and funded by the World Health Organization (Purchase Order 201710952). The Research Hub consists of several universities in Asia-Pacific countries. The authors of the paper appreciate technical and financial support from the Research Hub and the secretariat of APO in the completion of the project, upon which the paper was developed. The funders had no role in the design or conduct of the study; collection, management, analysis, and interpretation of the data; or preparation, review, and approval of the manuscript.

Conflicts of Interest: The authors declare they have no competing interests.

\section{Abbreviations}

$\begin{array}{ll}\text { CHW } & \text { Community health worker } \\ \text { CVDs } & \text { Cardiovascular diseases } \\ \text { THC } & \text { Township Health lefts } \\ \text { NCMS } & \text { New Cooperative Medical Scheme } \\ \text { NCDs } & \text { Non-communicable diseases } \\ \text { RCT } & \text { Randomized control trial } \\ \text { TB } & \text { Tuberculosis } \\ \text { VDs } & \text { Village doctors } \\ \text { WHO } & \text { World Health Organization }\end{array}$

\section{References}

1. Wang, L.; Kong, L.; Wu, F.; Bai, Y.; Burton, R. Preventing chronic diseases in China. Lancet 2005, 366, 1821-1824. [CrossRef]

2. World Health Organization (WHO). Noncommunicable Diseases. Country Profiles: China. Updated 2014. Available online: http:/ / www.who.int/nmh/countries/chn_en.pdf (accessed on 15 June 2017).

3. Wang, S.; Marquez, P.; Langenbrunner, J.; Niessen, L.; Suhrcke, M.; Song, F. Toward a healthy and harmonious life in China: Stemming the rising tide of non-communicable diseases. Available online: http://documents.worldbank.org/curated/en/618431468012000892/Toward-a-healthyand-harmonious-life-in-China-stemming-the-rising-tide-of-non-communicable-diseases (accessed on 20 September 2018).

4. Tang, S.; Ehiri, J.; Long, Q. China's biggest, most neglected health challenge: Non-communicable diseases. Infect. Dis. Poverty 2013, 2, 7. [CrossRef] [PubMed]

5. Qi, X.; Zhang, P.; Zhang, Y.; Wan, X.; Yang, G. China's prevention and control strategies on non-communicable diseases: The status quo. Chin. J. Prev. Cont. Chron. Non-Commun. Dis. 2012, 20, 214-217. (In Chinese)

6. Lewin, S.; Munabi-Babigumira, S.; Glenton, C.; Daniels, K.; Bosch-Capblanch, X.; van Wyk, B.E.; Odgaard-Jensen, J.; Johansen, M.; Aja, G.N.; Zwarenstein, M.; et al. Lay health workers in primary and community health care for maternal and child health and the management of infectious diseases. Cochrane Database Syst. Rev. 2010. [CrossRef] [PubMed]

7. World Health Organization (WHO). The World Health Report 2006: Working Together for Health. Available online: http:/ / apps.who.int/iris/handle/10665/43432 (accessed on 15 January 2018).

8. Sidel, V.W. The barefoot doctors of the People's Republic of China. N. Engl. J. Med. 1972, 286, $1292-1300$. [CrossRef] [PubMed]

9. de Geyndt, W.; Zhao, X.; Liu, S. From Barefoot Doctor to Village Doctor in Rural China; World Bank Technical Paper; The World Bank: Washington, DC, USA, 1992; Available online: http:/ /www.popline.org/node/ 324436 (accessed on 15 January 2018).

10. Wang, J.; Kushner, K.; Frey, J.J.; Du, X.P.; Qian, N. Primary care reform in the Peoples' Republic of China: Implications for training family physicians for the world's largest country. Fam. Med.-Kansas City 2007, $39,639$. 
11. Wang, Y.; Wilkinson, M.; Ng, E.; Cheng, K.K. Primary care reform in China. Br. J. Gen. Pract. 2012, 62, 546-547. [CrossRef] [PubMed]

12. Cherrington, A.; Ayala, G.X.; Amick, H.; Allison, J.; Corbie-Smith, G.; Scarinci, I. Implementing the community health worker model within diabetes management: Challenges and lessons learned from programs across the United States. Diabetes Educ. 2008, 34, 824-833. [CrossRef] [PubMed]

13. Browning, C.; Chapman, A.; Yang, H.; Liu, S.; Zhang, T.; Enticott, J.C.; Thomas, S.A. Management of type 2 diabetes in China: The Happy Life Club, a pragmatic cluster randomised controlled trial using health coaches. BMJ Open 2016, 6, e009319. [CrossRef] [PubMed]

14. Chai, J.; Shen, X.; Feng, R.; Cheng, J.; Chen, Y.; Zha, Z.; Jia, S.; Liang, H.; Zhao, T.; Sha, R.; et al. eCROPS-CA: A systematic approach toward effective and sustainable cancer prevention in rural China. BMC Cancer 2015, 15, 233. [CrossRef] [PubMed]

15. Tian, M.; Ajay, V.S.; Dunzhu, D.; Hameed, S.S.; Li, X.; Liu, Z.; Li, C.; Chen, H.; Cho, K.; Li, R.; et al. A cluster-randomized controlled trial of a simplified multifaceted management program for individuals at high cardiovascular risk (SimCard Trial) in rural Tibet, China and Haryana, India. Circulation 2015, 132, 815-824. [CrossRef] [PubMed]

16. Robinson, K.A.; Saldanha, J.; Mckoy, N.A. Development of a framework to identify research gaps from systematic reviews. J. Clin. Epidemiol. 2011, 64, 1325-1330. [CrossRef] [PubMed]

17. Moher, D.; Liberati, A.; Tetzlaff, J.; Altman, D.G.; Group, P. Preferred reporting items for systematic reviews and meta-analyses: The PRISMA statement. PLoS Med. 2009, 6, e1000097. [CrossRef] [PubMed]

18. Feng, R.; Li, K.; Cheng, J.; Xie, S.; Chai, J.; Wei, P.; Wang, D. Toward integrated and sustainable prevention against diabetes in rural China: Study rationale and protocol of eCROPS. BMC Endocr. Disord. 2013, 13, 28. [CrossRef] [PubMed]

19. Lin, C.W.; Abdullah, A.S.; Clinciu, D.L.; Scholl, J.; Jin, X.; Lu, H.; Chen, S.S.; Iqbal, U.; Heineck, M.J.; Li, Y.C. Empowering village doctors and enhancing rural healthcare using cloud computing in a rural area of mainland China. Comput. Methods Programs Biomed. 2014, 113, 585-592. [CrossRef] [PubMed]

20. Chen, P.; Chai, J.; Cheng, J.; Li, K.; Xie, S.; Liang, H.; Shen, X.; Feng, R.; Wang, D. A smart web aid for preventing diabetes in rural China: Preliminary findings and lessons. J. Med. Internet Res. 2014, 16, e98. [CrossRef] [PubMed]

21. Zhong, X.; Wang, Z.; Fisher, E.B.; Tanasugarn, C. Peer support for diabetes management in primary care and community settings in Anhui Province, China. Ann. Fam. Med. 2015, 13 (Suppl. 1), S50-S58. [CrossRef] [PubMed]

22. Li, T.; Lei, T.; Xie, Z.; Zhang, T. Determinants of basic public health services provision by village doctors in China: Using non-communicable diseases management as an example. BMC Health Serv. Res. 2016, 16, 42. [CrossRef] [PubMed]

23. Peiris, D.; Sun, L.; Patel, A.; Tian, M.; Essue, B.; Jan, S.; Zhang, P. Systematic medical assessment, referral and treatment for diabetes care in China using lay family health promoters: Protocol for the SMARTDiabetes cluster randomised controlled trial. Implement. Sci. 2016, 11, 116. [CrossRef] [PubMed]

24. Belinson, J.L.; Wang, G.; Qu, X.; Du, H.; Shen, J.; Xu, J.; Zhong, L.; Yi, J.; Yi, X.; Wu, R. The development and evaluation of a community based model for cervical cancer screening based on self-sampling. Gynecol. Oncol. 2014, 132, 636-642. [CrossRef] [PubMed]

25. Prince, M.; Ferri, C.P.; Acosta, D.; Albanese, E.; Arizaga, R.; Dewey, M.; Gavrilova, S.I.; Guerra, M.; Huang, Y.; Jacob, K.S.; et al. The protocols for the 10/66 dementia research group population-based research programme. BMC Public Health 2007, 7, 165. [CrossRef] [PubMed]

26. Gong, W.; Xu, D.; Zhou, L.; Brown III, H.S.; Smith, K.L.; Xiao, S. Village doctor-assisted case management of rural patients with schizophrenia: Protocol for a cluster randomized control trial. Implement. Sci. 2014, 9, 13. [CrossRef] [PubMed]

27. Chen, M.; Wu, G.; Wang, Z.; Yan, J.; Zhou, J.; Ding, Y.; Jiang, Y.; Rao, S.; Zhou, Q.; Ni, H.; et al. Two-year prospective case-controlled study of a case management program for community-dwelling individuals with schizophrenia. Shanghai Arch. Psychiatry 2014, 26, 119. [CrossRef] [PubMed]

28. Zhou, B.; Gu, Y. Effect of self-management training on adherence to medications among community residents with chronic schizophrenia: A singleblind randomized controlled trial in Shanghai, China. Shanghai Arch. Psychiatry 2014, 26, 332-338. [CrossRef] [PubMed] 
29. Ma, Z.; Huang, H.; Chen, Q.; Chen, F.; Abdullah, A.S.; Nie, G.; Feng, Q.; Wei, B. Mental health services in rural China: A qualitative study of primary health care providers. BioMed. Res. Int. 2015, 2015. [CrossRef] [PubMed]

30. Tang, X.; Yang, F.; Tang, T.; Yang, X.; Zhang, W.; Wang, X.; Ji, L.; Xiao, Y.; Ma, K.; Wang, Y.; et al. Advantages and challenges of a village doctor-based cognitive behavioral therapy for late-life depression in rural China: A qualitative study. PLoS ONE 2015, 10, e0137555. [CrossRef] [PubMed]

31. Xu, D.R.; Gong, W.; Caine, E.D.; Xiao, S.; Hughes, J.P.; Ng, M.; Simoni, J.; He, H.; Smith, K.L.; Brown III, H.S.; et al. Lay health supporters aided by a mobile phone messaging system to improve care of villagers with schizophrenia in Liuyang, China: Protocol for a randomised control trial. BMJ Open 2016, 6, e010120. [CrossRef] [PubMed]

32. Ajay, V.S.; Tian, M.; Chen, H.; Wu, Y.; Li, X.; Dunzhu, D.; Ali, M.K.; Tandon, N.; Krishnan, A.; Prabhakaran, D.; et al. A cluster-randomized controlled trial to evaluate the effects of a simplified cardiovascular management program in Tibet, China and Haryana, India: Study design and rationale. BMC Public Health 2014, 14, 924. [CrossRef] [PubMed]

33. Yan, L.L.; Fang, W.; Delong, E.; Neal, B.; Peterson, E.D.; Huang, Y.; Sun, N.; Yao, C.; Li, X.; MacMahon, S.; et al. Population impact of a high cardiovascular risk management program delivered by village doctors in rural China: Design and rationale of a large, cluster-randomized controlled trial. BMC Public Health 2014, 14, 345. [CrossRef] [PubMed]

34. Li, N.; Yan, L.L.; Niu, W.; Yao, C.; Feng, X.; Zhang, J.; Shi, J.; Zhang, Y.; Zhang, R.; Hao, Z.; et al. The effects of a community-based sodium reduction program in rural China-A cluster-randomized trial. PLoS ONE 2016, 11, e0166620. [CrossRef] [PubMed]

35. Chang, A.; Patberg, E.; Cueto, V.; Li, H.; Singh, B.; Kenya, S.; Alonzo, Y.; Carrasquillo, O. Community health workers, access to care, and service utilization among Florida Latinos: A randomized controlled trial. Am. J. Public Health. 2018, 108, 1249-1251. [CrossRef] [PubMed]

36. Ursua, R.A.; Aguilar, D.E.; Wyatt, L.C.; Trinh-Shevrin, C.; Gamboa, L.; Valdellon, P.; Perrella, E.G.; Dimaporo, M.Z.; Nur, P.Q.; Tandon, S.D.; et al. A community health worker intervention to improve blood pressure among Filipino Americans with hypertension: A randomized controlled trial. Prev. Med. Rep. 2018, 11, 42-48. [CrossRef] [PubMed]

37. Khetan, A.K.; Purushothaman, R.; Chami, T.; Hejjaji, V.; Madan Mohan, S.K.; Josephson, R.A.; Webel, A.R. The effectiveness of community health workers in CVD prevention in LMIC. Glob. Heart 2016, 12, 233-243. [CrossRef] [PubMed]

38. Perry, H.B.; Zulliger, R.; Rogers, M.M. Community health workers in low-, middle-, and high-income countries: An overview of their history, recent evolution, and current effectiveness. Ann. Rev. Public Health 2014, 35, 399-421. [CrossRef] [PubMed]

39. World Health Organization (WHO). Global Health Observatory Data: Risk Factors. 2017. Available online: http:/ / www.who.int/gho/ncd/risk_factors/en/ (accessed on 15 January 2018).

40. Koon, A.D.; Goudge, J.; Norris, S.A. A review of generalist and specialist community health workers for delivering adolescent health services in sub-Saharan Africa. Hum. Resour. Health 2013, 11, 54. [CrossRef] [PubMed]

41. Reininger, B.M.; Mitchell-Bennett, L.; Lee, M.; Gowen, R.Z.; Barroso, C.S.; Gay, J.L.; Saldana, M.V. Tu Salud, ¡Si Cuenta!: Exposure to a community-wide campaign and its associations with physical activity and fruit and vegetable consumption among individuals of Mexican descent. Soc. Sci. Med. 2015, 143, 98-106. [CrossRef] [PubMed]

42. Shelley, D.; VanDevanter, N.; Cleland, C.C.; Nguyen, L.; Nguyen, N. Implementing tobacco use treatment guidelines in community health centers in Vietnam. Implement. Sci. 2015, 10, 142. [CrossRef] [PubMed]

43. Wewers, M.E.; Shoben, A.; Conroy, S.; Curry, E.; Ferketich, A.K.; Murray, D.M.; Nemeth, J.; Wermert, A. Effectiveness of two community health worker models of tobacco dependence treatment among community residents of Ohio Appalachia. Nicotine Tob. Res. 2017, 19, 1499-1507. [PubMed]

44. Abrahams-Gessel, S.; Denman, C.A.; Gaziano, T.A.; Levitt, N.S.; Puoane, T. Challenges facing successful scaling up of effective screening for cardiovascular disease by community health workers in Mexico and South Africa: Policy implications. Health Syst. Policy Res. 2016, 3, 26.

45. Zhang, X.; Fang, P. Job satisfaction of village doctors during the new healthcare reforms in China. Aust. Health Rev. 2016, 40, 225-233. [CrossRef] [PubMed] 
46. Gaziano, T.A.; Abrahams-Gessel, S.; Denman, C.A.; Montano, C.M.; Khanam, M.; Puoane, T.; Levitt, N.S. An assessment of community health workers' ability to screen for cardiovascular disease risk with a simple, non-invasive risk assessment instrument in Bangladesh, Guatemala, Mexico, and South Africa: An observational study. Lancet Glob. Health 2015, 3, e556-563. [CrossRef]

47. Han, H.R.; Kim, K.B.; Kim, M.T. Evaluation of the training of Korean community health workers for chronic disease management. Health Educ. Res. 2007, 22, 513-521. [CrossRef] [PubMed]

48. Asiimwe-Kateera, B.; Condo, J.; Ndagijimana, A.; Kumar, S.; Mukeshimana, M.; Gaju, E.; Muhire, A.; Muhimpundu, M.A.; Kim, M.J.; Kurth, A. Analysis: Mobile health approaches to non-communicable diseases in Rwanda. Rwanda J. 2015, 2, 89-92. [CrossRef]

49. DiCarlo, J.M.; Gopakumar, S.; Dhillon, P.K.; Krishnan, S. Adoption of Information and communication technologies for early detection of breast and cervical cancers in low-and middle-income countries. J. Glob. Oncol. 2016, 2, 222-234. [CrossRef] [PubMed]

50. Amarchand, R.; Sharma, H.; Krishnan, A. Community health workers can be trained to measure blood pressure: Experience from India. Reg. Health Forum. 2013, 17, 26-31.

51. Wagstaff, A.; Lindelow, M.; Jun, G.; Ling, X.; Juncheng, Q. Extending health insurance to the rural population: An impact evaluation of China's new cooperative medical scheme. J. Health Econ. 2009, 28, 1-19. [CrossRef] [PubMed]

52. Wang, Q.; Wang, W.; Yan, F. Compensation and welfare of village doctors under integrated management of township and village health facilities. Chin. Rural Health Serv. Adm. 2011, 31, 7-9.

53. Zhang, S.; Zhang, W.; Zhou, H.; Xu, H.; Qu, Z.; Guo, M.; Wang, F.; Zhong, Y.; Gu, L.; Liang, X.; et al. How China's new health reform influences village doctors' income structure: Evidence from a qualitative study in six counties in China. Hum. Resour. Health 2015, 13, 26. [CrossRef] [PubMed]

54. Kok, M.C.; Kane, S.S.; Tulloch, O.; Ormel, H.; Theobald, S.; Dieleman, M.; Taegtmeyer, M.; Broerse, J.E.; de Koning, K.A. How does context influence performance of community health workers in low- and middle-income countries? Evidence from the literature. Health Res. Policy Syst. 2015, 13, 13. [CrossRef] [PubMed]

55. Kasteng, F.; Settumba, S.; Källander, K.; Vassall, A. Valuing the work of unpaid community health workers and exploring the incentives to volunteering in rural Africa. Health Policy Plan. 2016, 31, 205-216. [CrossRef] [PubMed]

56. Mpembeni, R.N.M.; Bhatnagar, A.; LeFevre, A.; Chitama, D.; Urassa, D.P.; Kilewo, C.; Mdee, R.M.; Semu, H.; Winch, P.J.; Killewo, J.; et al. Motivation and satisfaction among community health workers in Morogoro Region, Tanzania: Nuanced needs and varied ambitions. Hum. Resour. Health 2015, 13, 44. [CrossRef] [PubMed]

57. Yip, W.C.M.; Hsiao, W.C.; Chen, W.; Hu, S.; Ma, J.; Maynard, A. Early appraisal of China's huge and complex health-care reforms. Lancet 2012, 379, 833-842. [CrossRef]

58. Zhou, X.D.; Li, L.; Hesketh, T. Health system reform in rural China: Voices of healthworkers and service-users. Soc. Sci. Med. 2014, 117, 134-141. [CrossRef] [PubMed]

59. Zhang, L.; Wang, Z.; Qian, D.; Ni, J. Effects of changes in health insurance reimbursement level on outpatient service utilization of rural diabetics: Evidence from Jiangsu Province, China. BMC Health Serv. Res. 2014, 14, 185. [CrossRef] [PubMed]

60. Jafar, T.H.; Islam, M.; Hatcher, J.; Hashmi, S.; Bux, R.; Khan, A.; Poulter, N.; Badruddin, S.; Chaturvedi, N. Community based lifestyle intervention for blood pressure reduction in children and young adults in developing country: Cluster randomised controlled trial. BMJ 2010, 340, c2641. [CrossRef] [PubMed]

61. Coleman, R.; Gill, G.; Wilkinson, D. Noncommunicable disease management in resource-poor settings: A primary care model from rural South Africa. Bull. World Health Organ. 1998, 76, 633. [PubMed]

62. Van Rie, A.; West, N.S.; Schwartz, S.R.; Mutanga, L.; Hanrahan, C.F.; Ncayiyana, J.; Bassett, J. The unmet needs and health priorities of the urban poor: Generating the evidence base for urban community health worker programmes in South Africa. S. Afr. Med. J. 2018, 108, 734-740. [CrossRef] [PubMed]

(C) 2018 by the authors. Licensee MDPI, Basel, Switzerland. This article is an open access article distributed under the terms and conditions of the Creative Commons Attribution (CC BY) license (http:// creativecommons.org/licenses/by/4.0/). 